Preprint typeset in JHEP style - HYPER VERSION

hep-th/0606078

\title{
From Spacetime to Worldsheet: Four point correlators
}

\author{
Justin R. David, Rajesh Gopakumar \\ Harish-Chandra Research Institute, \\ Chhatnag Road., Jhunsi, \\ Allahabad 211019, India. \\ justin, gopakumr@hri.res.in
}

\begin{abstract}
The Schwinger representation gives a systematic procedure for recasting large $N$ field theory amplitudes as integrals over closed string moduli space. This procedure has recently been applied to a class of free field four point functions by Aharony, Komargodski and Razamat, to study the leading terms in the putative worldsheet OPE. Here we observe that the dictionary between Schwinger parameters and the cross ratio of the four punctured sphere actually yields an explicit expression for the full worldsheet four point correlator in many such cases. This expression has a suggestive form and obeys various properties, such as crossing symmetry and mutual locality, expected of a correlator in a two dimensional CFT. Therefore one may take this to be a candidate four point function in a worldsheet description of closed strings on highly curved $A d S_{5} \times S^{5}$. The general framework, that we develop for computing the relevant Strebel differentials, also admits a systematic perturbation expansion which would be useful for studying more general four point correlators.
\end{abstract}




\section{Contents}

1. Introduction 1

2. Strebel differentials and the four punctured sphere 6

2.1 Strebel differentials and Schwinger parameters 6

2.2 Strebel differentials for the four-punctured sphere 0

3. The Strebel differential for the $Y$ diagram 11

4. A worldsheet four point function 14

5. A perturbation scheme around the $Y$ diagram 18

6. Conclusions 22

A. Elliptic Functions 23

B. Strebel differential in terms of Weierstrass functions 27

C. The elementary derivation of equation (3.12) 31

D. Details on the approximation scheme 32

E. Solution for the modulus $k$ in terms of the Strebel lengths. 36

\section{Introduction}

One of the major hurdles, which repeatedly crops up, in the study of the AdS/CFT conjecture [1, 2, 3] is the lack of a complete worldsheet description of the closed string theory on $A d S_{5} \times S^{5}$. The conventional RNS formulation is not of much use because of the presence of RR flux in the background. The Green-Schwarz approach, on the other hand, has the problem of requiring some kind of lightcone gauge fixing to

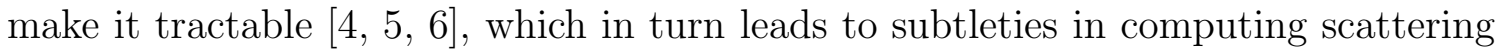
amplitudes. There also exists a covariant formulation of the worldsheet theory due to Berkovits [7, 8], but it has not been developed to the stage where we can use it for 
comparisons with gauge theory calculations, specially in the perturbative domain of weak 'tHooft coupling'.

Perhaps, what these difficulties are pointing to, is that quantising the worldsheet theory of strings in these backgrounds may be a bit like quantising Liouville theory (For a review, see [29]). The path integral (as well as the operator) approach have yielded limited information about Liouville theory. The most complete description, yet, has come from the algebraic approach - the so-called conformal bootstrap. This came in the form of inspired conjectures for the three point functions [30, 31]. It was then understood that the crossing symmetry constraints on the structure constants, coming from four point functions, was sufficent for a purely algebraic determination [32, 33.

In like manner, we could aim for a purely algebraic characterisation of the full worldsheet CFT (with ghosts and/or other auxiliary fields) for $A d S_{5} \times S^{5}$. The four point functions of this $c=0 \mathrm{CFT}$ would then contain the information necessary to characterise the theory. By factorising in different channels one can read off the structure constants $C_{i j k}$ and the structure of the conformal blocks.

How can we obtain these four point functions of the worldsheet CFT, without much knowledge about the sigma model? A concrete prescription to implement openclosed string duality for large $N$ gauge theories has been given in [34, 35, 36, 37]. Essentially, one recasts field theory correlation functions, in Schwinger parametrisation, as integrals over the closed string moduli space of worldsheet correlators (see also [38, 39, 40, 41, 42 for some further explorations). In particular, one can study the planar four point function of gauge invariant operators in the free gauge theory (as a starting point for a perturbative expansion in the 't Hooft coupling $\lambda$ ). The prescription of [35, 37] enables one to rewrite the simple spacetime answer as an integral over the cross ratio that parametrises the four punctured sphere. The integrand is then a natural candidate ${ }^{2}$ for the four point function of the corresponding vertex

\footnotetext{
${ }^{1}$ Many different approaches have been taken to study the string dual of the weakly coupled gauge theory. For a light cone approach, see 19, 10, 11, 12, 13, 14. For a string bit approach, see [15, 16, 17, 18] and also from a discretised worldsheet point of view [19, 20, 21, 22, 23, 24, 25]. See also [26, 27, 28].

${ }^{2}$ The answer given by this prescription is presumably that obtained by fixing the worldsheet diffeomorphisms to the locally flat metric given by the Strebel construction of the Riemann surface
} 
operators in the worldsheet CFT. It is, in fact, a nontrivial check of the proposal that the integrand thus obtained (expressed in terms of the cross ratio) even satisfies all the generic properties one might expect of a worldsheet correlator in a local $2 \mathrm{~d}$ CFT. This is certainly not guaranteed by the construction.

There is, however, a technical hurdle to be overcome in implementing and checking this proposal. Generically, the change of variables between the Schwinger parameters and the usual complex parameters on moduli space (such as the cross ratio, in the case of the four punctured sphere) is given by a transcendental relation [37] which makes it hard to obtain explicit expressions for the worldsheet correlator. Therefore one strategy is to look at special correlators for which there is a simplification.

In [43] four point correlation functions of the form

$$
\Gamma_{\left\{J_{i}\right\}}^{(4)}\left(x_{1}, x_{2}, x_{3}\right)=\left\langle\operatorname{Tr} \Phi^{J_{1}}\left(x_{1}\right) \operatorname{Tr} \Phi^{J_{2}}\left(x_{2}\right) \operatorname{Tr} \Phi^{J_{3}}\left(x_{3}\right) \operatorname{Tr} \Phi^{J}(0)\right\rangle
$$

were considered (with $J=J_{1}+J_{2}+J_{3}$ and $\Phi$ being an adjoint field under the $U(N)$ gauge group). In these cases, because of the simpler nature of the free field contractions it is possible to be much more explicit. In fact, Aharony et. al 43 considered the contribution to (1.1) from the $Y$ shaped diagram of free field theory and used the change of variables to study the behavior of the candidate worldsheet correlator when one of the punctures approaches another. In other words, they looked at the leading (and next to leading) behaviour in the worldsheet OPE. They found that for both these terms the behaviour was consistent with what might be expected of a worldsheet correlator in a string theory. Though the worldsheet conformal weights $h, \bar{h}$ could be individually half integral, the difference $(h-\bar{h})$ was an integer, at least to this order.

In this paper, we will develop a general method for obtaining the change of variables from the Schwinger parameters of a generic free field four point function to the cross ratio $\eta$. Since the dictionary between the Schwinger parameters and $\eta$ is through Strebel quadratic differentials on the four punctured sphere, we need to study the latter. Generically the expressions will involve elliptic functions. However, as expected, things simplify for the special class of $Y$ shaped Feynman graphs studied (see [37]). Of course, one may always add total derivatives on moduli space to the answer obtained this way. 
by Aharony et.al. [43]. On taking a particular limit, our method recovers their results. In fact, by working directly in terms of the cross ratio $\eta$ we are able to find a completely explicit expression for the corresponding worldsheet correlator.

To relate to results for $\mathcal{N}=4$ Yang-Mills theory, we could study the analogue of (1.1) which is

$$
\tilde{\Gamma}_{\left\{J_{i}\right\}}^{(4)}\left(x_{1}, x_{2}, x_{3}\right)=\left\langle\operatorname{Tr} Z^{J_{1}}\left(x_{1}\right) \operatorname{Tr} Z^{J_{2}}\left(x_{2}\right) \operatorname{Tr} Z^{J_{3}}\left(x_{3}\right) \operatorname{Tr} \bar{Z}^{J}(0)\right\rangle
$$

Here and below $X, Y, Z$ will denote the complex scalars in the three chiral multiplets of $\mathcal{N}=4$ Yang-Mills theory. With $J=J_{1}+J_{2}+J_{3}$, (1.2) is an example of a socalled extremal correlator [44] $]^{3}$. In fact, these are a particularly interesting class of correlators to study from the point of view of the AdS/CFT conjecture since they are not renormalised from their free field value. This is a generalisation of the nonrenormalisation theorem for three point functions [47]. The $Y$ shaped diagram is one of the contributions to this correlator in the free theory and thus the expression we derive is of relevance for this case. However, there are other diagrams that need to be taken into account as well, in evaluating the complete worldsheet correlator ${ }^{4}$.

However, we can readily give examples of spacetime correlators which gets contributions in the free $\mathcal{N}=4$ Yang-Mills theory only from the $Y$ shaped graph.

$$
\Gamma_{\left\{J_{i}\right\}}^{(4)}\left(x_{1}, x_{2}, x_{3}\right)=\left\langle\operatorname{Tr} X^{J_{1}}\left(x_{1}\right) \operatorname{Tr} Y^{J_{2}}\left(x_{2}\right) \operatorname{Tr} Z^{J_{3}}\left(x_{3}\right) \operatorname{Tr}\left(\bar{X}^{J_{1}} \bar{Y}^{J_{2}} \bar{Z}^{J_{3}}\right)(0)\right\rangle
$$

The operators entering here are not all chiral primaries unlike in (1.2). The last operator is an admixture of stringy and supergravity modes in the dual theory. We can use the change of variables mentioned above to express this correlator as a worldsheet integral

$$
\Gamma_{\left\{J_{i}\right\}}^{(4)}\left(x_{1}, x_{2}, x_{3}\right)=\int d^{2} \eta G_{\left\{x_{i}\right\}}^{\left\{J_{i}\right\}}(\eta, \bar{\eta}) .
$$

One of the results of this paper is that the above change of variables applied to this amplitude yields an explicit expression for the candidate worldsheet correlator. $G_{\left\{x_{i}\right\}}^{\left\{J_{i}\right\}}(\eta, \bar{\eta})$ in $(1.3)$

\footnotetext{
${ }^{3}$ There is a large amount of AdS/CFT literature on four point functions of various operators. See the reviews 445] 46] for references.

${ }^{4}$ We are grateful to O. Aharony, Z. Komargodski and S. Razamat for pointing this out to us. The contribution of these additional diagrams are currently being evaluated 48].
} 


$$
\begin{aligned}
& G_{\left\{x_{i}\right\}}^{\left\{J_{i}\right\}}(\eta, \bar{\eta})=C\left(J_{i}\right) \frac{(1+|\eta|+|1-\eta|)^{\frac{1}{2}}}{|\eta||1-\eta|} \times \\
& \frac{(1-|\eta|+|1-\eta|)^{J_{1}-\frac{1}{2}}(1+|\eta|-|1-\eta|)^{J_{2}-\frac{1}{2}}(-1+|\eta|+|1-\eta|)^{J_{3}-\frac{1}{2}}}{\left[x_{1}^{2}(1-|\eta|+|1-\eta|)+x_{2}^{2}(1+|\eta|-|1-\eta|)+x_{3}^{2}(-1+|\eta|+|1-\eta|)\right]^{J}} .
\end{aligned}
$$

If we are to make the identification

$$
G_{\left\{x_{i}\right\}}^{\left\{J_{i}\right\}}(\eta, \bar{\eta})=\left\langle\mathcal{V}_{x_{1}}^{J_{1}}(0) \mathcal{V}_{x_{2}}^{J_{2}}(1) \mathcal{V}_{x_{3}}^{J_{3}}(\infty) \overline{\mathcal{V}}_{x_{4}=0}^{\{J\}}(\eta, \bar{\eta})\right\rangle_{W S}
$$

with a worldsheet correlator of primary vertex operators, then an essential requirement is that $G_{\left\{x_{i}\right\}}^{\left\{J_{i}\right\}}(\eta, \bar{\eta})$ satisfy the crossing symmetry relations

$$
\begin{aligned}
G_{x_{2} x_{1} x_{3}}^{J_{2} J_{1} J_{3} J}(1-\eta, 1-\bar{\eta}) & =G_{x_{1} x_{2} x_{3}}^{J_{1} J_{2} J_{3} J}(\eta, \bar{\eta}) \\
G_{x_{3} x_{2} x_{1}}^{J_{3} J_{2} J_{1} J}\left(\frac{1}{\eta}, \frac{1}{\bar{\eta}}\right) & =|\eta|^{4} G_{x_{1} x_{2} x_{3}}^{J_{1} J_{2} J_{3} J}(\eta, \bar{\eta}) .
\end{aligned}
$$

As can be readily verified, $G_{\left\{x_{i}\right\}}^{\left\{J_{i}\right\}}(\eta, \bar{\eta})$ in (1.5) satisfies these relations. It is also consistent with locality as all the terms in the OPE (when $\eta \rightarrow 0$ )

$$
G_{\left\{x_{i}\right\}}^{\left\{J_{i}\right\}}(\eta, \bar{\eta})=\sum_{h, \bar{h}} C_{h, \bar{h}}^{J_{i}, x_{i}} \eta^{h} \bar{\eta}^{\bar{h}}
$$

have $(h-\bar{h})$ integral, even though the weights can be individually half integral. This generalises the results of [43], for the leading couple of terms, to all orders in the expansion.

The form of the correlator is also very suggestive with the dependence on $|\eta|$ and $|1-\eta|$ being what one might expect of a correlation function of local operators inserted at $0,1, \infty$ and $\eta$. As we will discuss in Sec.4, there is a relation of individual terms in this expression to the four point correlators of spin fields. This raises the possibility of understanding at least this class of correlators in an elementary way. It should be mentioned that the procedure by which (1.5) is obtained, readily generalises to any other correlator which gets a contribution from a $Y$ shaped diagram. One can easily write the corresponding worldsheet expression for any such gauge correlator in the $\mathcal{N}=4$ Yang-Mills theory, For that matter, the theory need not even be supersymmetric.

We will also go ahead and consider a systematic perturbation expansion around the limiting form of the Strebel differential we had employed in the above considerations. This expansion would be relevant to studying correlators, more general than 
the one considered above. The expansion is surprisingly non-trivial and needs to be done quite carefully. We will give partially explicit expressions for the perturbed cross ratio in terms of the Strebel lengths (which are identified with the Schwinger parameters). We will however postpone an application of this procedure to studying more general worldsheet correlators for the future.

The organisation of this paper is as follows. In the next section we review some general facts about Strebel differentials and in particular, the parametrisation of the differential for the four punctured sphere. We will then give the general procedure for obtaining the relation between the Schwinger parameters and the cross ratio. In section 3 , we show how this change of variables simplifies in a particular limit which corresponds to the $Y$ diagrams that contribute to the correlators defined above. We obtain the explicit relation between the cross ratio and the Schwinger parameters (see (3.12)). Those who would like to skip the various technicalities of Strebel differentials can directly go to (3.12). We use this relation in section 4. to study the Schwinger parametrised form of the correlator (1.3). We deduce the form (1.5) and comment on its various features as well as the clues it might give us about the worldsheet theory. In section 5., we give an algorithm for performing a systematic perturbation around the limiting differential of section 3. We obtain a well defined expansion in terms of the Strebel lengths in certain limits. Section 6. is a brief conclusion. Various appendices contain technical details that arise at different points in the main text.

\section{Strebel differentials and the four punctured sphere}

\subsection{Strebel differentials and Schwinger parameters}

The precise recipe for obtaining the closed string correlators from the Schwinger representation of the field theory amplitude is via a special kind of holomorphic quadratic differential $\phi(z) d z^{2}$ on the corresponding closed string Riemann surface. The properties of these Strebel differentials have been reviewed in [37, 43] and we refer the reader to these papers and references therein.

Very briefly, Strebel differentials have double poles at $n$ marked points (which will be identified with closed string vertex operator insertions). There is a critical graph (of genus $g$ with $n$ faces) associated to each such differential with each face of 
the critical graph enclosing a double pole of the Strebel differential. The vertices of this graph are the zeroes of the differential, with a vertex of valence $k$ associated to a zero of order $(k-2)$. The edges of the graph are the so-called non-closed horizontal trajectories along which $\sqrt{\phi(z)} d z$ is real. Therefore the Strebel lengths defined by

$$
l_{r}=\int_{e_{r}} \sqrt{\phi(z)} d z
$$

are real, where $e_{r}$ is an edge connecting two zeroes of the differential.

As can be guessed from the identification of the poles (in each face) with vertex operator insertions, the dual to the critical graph is associated with the field theory Feynman diagrams in the $A d S / C F T$ correspondence. More precisely, the skeleton diagram [35], obtained by gluing together homotopic Wick contractions in Feynman diagrams, is dual to the critical graph [37]. Thus, for a Feynman diagram of genus $g$ with $n$ vertices, we need to consider a Strebel differential on a Riemann surface of the same genus and with $n$ marked points. It is a theorem due to Strebel that given such a Riemann surface $\Sigma_{g, n} \in \mathcal{M}_{g, n}$ together with $n$ residues $\left\{p_{a}\right\}$ at the double poles, there is a unique Strebel differential. As a consequence, there is a one to one mapping between the $(3 g-3+n)$ complex moduli $\eta_{i}$ and $n$ residues $p_{a}$ to the $(6 g-6+3 n)$ Strebel lengths $l_{r}$. Summing over the inequivalent skeleton graphs of genus $g$ with $n$ vertices, and varying the lengths of the edges then gives a single cover of the decorated moduli space $\mathcal{M}_{g, n} \times R_{+}^{n}$

The proposal to implement open-closed duality is to identify the inverse Schwinger times 37

$$
\sigma_{r}=\frac{1}{\tau_{r}}=l_{r}=\int_{e_{r}} \sqrt{\phi\left(z, \eta_{i}, p_{a}\right)} d z .
$$

We use this to make a change of variables of the Schwinger integrand from the $\sigma_{r}$ to the complex moduli $\eta_{i}$ (as well as the residues $p_{a}$ ). Performing the integral over the $\left\{p_{a}\right\} \in R_{+}^{n}$ leaves us with an integral over $\mathcal{M}_{g, n}$ parametrised by the usual complex moduli $\eta_{i}$. It is the integrand here that we can take to be a candidate correlator of the worldsheet CFT.

\subsection{Strebel differentials for the four-punctured sphere}

Since we are interested in planar four point functions (in the free theory, to begin with) we will look at the Strebel differentials on the four punctured sphere. This can 
always be put in the following form

$$
\phi(z) d z^{2}=-C \frac{\left(z^{2}-1\right)\left(z^{2} k^{2}-1\right)}{\left(z-z_{0}\right)^{2}\left(z-z_{1}\right)^{2}\left(z-z_{2}\right)^{2}\left(z-z_{3}\right)^{2}} d z^{2} .
$$

Here we have chosen the double poles to be at at $z_{0}, z_{1}, z_{2}, z_{3}$ and used the freedom of $S L(2, C)$ transformations to put the zeros at $\pm 1, \pm 1 / k$. Both $C$ and $k$ are as yet undetermined complex contants. This parametrization of the Strebel differential assumes no additional symmetries. By Strebel's theorem, given the cross ratio $\eta$ of the punctures and the residues at the four punctures, the constants $C$ and $k$ as well as the locations of the poles $z_{i}$ are determined. Below we will see how this happens.

The u-plane

From (2.3), we see that $\int \sqrt{\phi(z)} d z$ would be most naturally expressed in terms of elliptic functions. Therefore we introduce the auxiliary $u$-plane where the doubly periodic properties of the differential $\sqrt{\phi(z)} d z$ are more manifest. With $w^{2}=\left(z^{2}-\right.$ 1) $\left(z^{2} k^{2}-1\right)$, we define the variable $u$ to be

$$
u=\int_{1}^{z} \frac{d z}{w} .
$$

Upto a constant shift this is essentially the defining relation for the Jacobi elliptic function $\operatorname{sn}(u)$ of modulus $k$. We have the relation

$$
z=\operatorname{sn}\left(u+\frac{1}{2} \omega_{1}\right)=\frac{\operatorname{cn}(u)}{\operatorname{dn}(u)},
$$

where $2 \omega_{1} \equiv 4 K(k)$ is one of the periods of $\operatorname{sn}(u)$ (with modulus $k$ ). Note that at $u=0, z=\operatorname{sn}(K)=1$ which fixes this integration constant. For future reference we note that the second period of the $\operatorname{sn}(u)$ function is given by $2 \omega_{2}=2 i K^{\prime}(k)$. In other words, the torus that the $u$-plane defines has periods $\left(2 \omega_{1}, 2 \omega_{2}\right)$. In appendix A. we gather together some basic facts (and notation) regarding elliptic functions which we will use.

We can now write the differential $\sqrt{\phi(z)} d z$ in the $u$-plane ${ }^{5}$

$$
\sqrt{\phi(u)} d u=-i \sqrt{C} k^{\prime 4} \frac{\operatorname{sn}^{2}(u)}{\prod_{i=0}^{3}\left(\operatorname{cn}(u)-z_{i} \operatorname{dn}(u)\right)} d u,
$$

\footnotetext{
${ }^{5}$ By abuse of notation we will use the same symbol $\phi$ for the $u$-plane as in the $z$-plane, though the functional form of $\phi$ is different in both cases.
} 
where $k^{\prime}=\sqrt{1-k^{2}}$. We have used various shift identities for the Jacobi functions (A.6) and the formulae for their derivatives (A.8) to arrive at this form. From (2.6), together with the locations of zeroes and poles of the Jacobi elliptic functions, we can see that $\sqrt{\phi(u)}$ has double zeros at $0, \omega_{1}, \omega_{2}, \omega_{1}+\omega_{2}$ (modulo the double periodicity $\left.\left(2 \omega_{1}, 2 \omega_{2}\right)\right)$. Furthermore from (2.6) it is easy to see that both $u_{i}$ and $-u_{i}$ (where $\left.\operatorname{cn}\left(u_{i}\right) / \operatorname{dn}\left(u_{i}\right)=z_{i} ; i=0 \ldots 3\right)$ are simple poles of $\sqrt{\phi(u)}$ with residues

$$
\begin{aligned}
r_{i} & =-i \sqrt{C} k^{\prime 2} \frac{\operatorname{sn}\left(u_{i}\right) \operatorname{dn}\left(u_{i}\right)}{\prod_{j \neq i}\left(\operatorname{cn}\left(u_{i}\right)-z_{j} \operatorname{dn}\left(u_{i}\right)\right)}, \\
& =-i \sqrt{C} k^{\prime 2} \frac{\operatorname{sn}\left(u_{i}\right) \prod_{k=0}^{3} \operatorname{dn}\left(u_{k}\right)}{\prod_{j \neq i}\left(\operatorname{cn}\left(u_{i}\right) \operatorname{dn}\left(u_{j}\right)-\operatorname{cn}\left(u_{j}\right) \operatorname{dn}\left(u_{i}\right)\right)} .
\end{aligned}
$$

Note that these properties on the $u$-plane (double zeroes and simple poles) are not in contradiction with the different behaviour on the $z$-plane since this auxiliary torus is a branched cover of the original sphere.

We would like to determine the positions of the poles $z_{i}$ (or $u_{i}$ ) and thus their cross ratio, in terms of the $r_{i}$. Either by writing the Strebel differential as

$$
\sqrt{\phi(u)} d u \propto \sum_{i=0}^{3} r_{i} \frac{\operatorname{sn}\left(u_{i}\right)}{\operatorname{dn}\left(u_{i}\right)^{2}} \frac{\operatorname{dn}(u)}{\operatorname{cn}(u)-z_{i} \operatorname{dn}(u)},
$$

and demanding that it have the right double zeroes or else from direct verification one has the relations

$$
\begin{aligned}
\sum_{i} r_{i} \frac{1}{\operatorname{sn}\left(u_{i}\right)} & =0, \\
\sum_{i} r_{i} \operatorname{sn}\left(u_{i}\right) & =0, \\
\sum_{i} r_{i} \frac{\operatorname{cn}\left(u_{i}\right) \operatorname{dn}\left(u_{i}\right)}{\operatorname{sn}\left(u_{i}\right)} & =0 .
\end{aligned}
$$

In fact, if we substitute $r_{i}$ given in (2.7) into (2.9) then those three equations are equivalent to the algebraic identities

$$
\begin{aligned}
& \sum_{i} \frac{1}{\prod_{j \neq i}\left(z_{i}-z_{j}\right)}=0, \\
& \sum_{i} \frac{z_{i}}{\prod_{j \neq i}\left(z_{i}-z_{j}\right)}=0, \\
& \sum_{i} \frac{z_{i}^{2}}{\prod_{j \neq i}\left(z_{i}-z_{j}\right)}=0,
\end{aligned}
$$


where $z_{i}=\operatorname{cn}\left(u_{i}\right) / d n\left(u_{i}\right)$. In principle the equations (2.9) determine three of the four $u_{i}$ in terms of the fourth, say $u_{0}$, as well as the $r_{i}$. To determine $u_{0}$ we also need to know the Strebel lengths between zeroes.

\section{The Strebel lengths}

The main reason to go to the $u$-plane is that it enables us carry out the Strebel integrals between the zeroes. The two independent lengths can be taken to be

$$
a=\int_{0}^{\omega_{1}} \sqrt{\phi(u)} d u
$$

and

$$
b=-\int_{0}^{\omega_{2}} \sqrt{\phi(u)} d u .
$$

To carry out these integrals it will be useful to use an alternative representation of the Strebel differential in terms of the Weierstrass functions (see Appendix A.)

$$
\sqrt{\phi(u)} d u=i \sum_{i} r_{i}\left(\zeta\left(u+u_{i}\right)-\zeta\left(u-u_{i}\right)-2 \zeta\left(u_{i}\right)\right) d u
$$

By the properties of $\zeta(u)$, we can see that this has simple poles at $u= \pm u_{i}$ with residue $\pm r_{i}$. It can also be verified (as shown in appendix B.) that this has the right double zeroes if the relations (2.9) hold. From the uniqueness properties of elliptic functions this is sufficient to conclude that (2.13) is the same as (2.6).

Using the fact that $\zeta(u)$ is the derivative of a quasiperiodic function, we can easily evaluate the integrals in equations (2.11),(2.12) to obtain

$$
\begin{aligned}
a & =\sum_{i} r_{i}\left[\pi-2 i\left(\zeta\left(u_{i}\right) \omega_{1}-\zeta\left(\omega_{1}\right) u_{i}\right)\right] \\
b & =\sum_{i} r_{i}\left[\pi+2 i\left(\zeta\left(u_{i}\right) \omega_{2}-\zeta\left(\omega_{2}\right) u_{i}\right)\right] .
\end{aligned}
$$

For the future we will also record a useful linear combination of these two equations.

$$
\pi \sum_{i} r_{i} u_{i}=\left(\pi \omega_{1}+\pi \omega_{2}\right) \sum_{i} r_{i}-a \omega_{2}-b \omega_{1}
$$

where we have used the Legendre relation $2\left(\omega_{2} \zeta\left(\omega_{1}\right)-\omega_{2} \zeta\left(\omega_{2}\right)\right)=i \pi$.

The strategy we will adopt is to assume one is given the residues $r_{i}$ and the Strebel lengths $a, b$ - these are after all linear combinations of Schwinger parameters. 
It is clear from (2.9) we can eliminate all the poles $u_{1}, u_{2}, u_{3}$ in terms of $u_{0}$. Then from the two real equations (2.14) we can, in principle, determine the pole $u_{0}$ in terms of $a, b, r_{i}$. Thus the cross ratios of the poles $z_{i}=\frac{\operatorname{cn} u_{i}}{\operatorname{dn} u_{i}}$ is determined in terms of $r_{i}$ and $(a, b)$ as required. In practice, this is a difficult task to carry out explicitly.

\section{The Strebel differential for the $Y$ diagram}

Aharony et. al. 43 made the nice observation that the Strebel differential corresponding to a $Y$ shaped skeleton Feynman diagram is quite simple. Moreover, this simple differential exists for every point on the moduli space of the four punctured sphere. In other words, as we vary the Schwinger parameters for the $Y$ diagram, we cover the entire moduli space and not just some subspace. In this section, we will look at this simple Strebel differential from the point of view of the general framework outlined in the previous section. While this is not necessary for obtaining the final result (3.12), which can be obtained by simpler methods, the general framework will be useful in developing a systematic perturbation expansion around this simple differential. For completeness, in appendix C., we will outline the elementary way of obtaining the solution which is essentially equivalent to the way in which it was solved in 443].

The simplification of the $Y$ diagram is that the dual graph has three edges, one vertex and four faces. In fact, the valency of the vertex is six corresponding to a fourth order zero for the differential, as per the general properties mentioned in section 2.1. Therefore instead of elliptic functions, $\sqrt{\phi(z)}$ is algebraic and we can easily solve for the Strebel conditions 43] (See also appendix C.). The fourth order zero is clearly a limit of the separated zeroes in a generic Strebel differential. How do we take this limit in the general differential of the form (2.3)? Notice that if the

poles $z_{i}=\frac{z_{i}^{\prime}}{\epsilon}$ with $z_{i}^{\prime}$ finite and $\epsilon \rightarrow 0$, then it would be natural to make the scaling of the variable $z=\frac{z^{\prime}}{\epsilon}$ in (2.3). In other words we are taking a large $z$ limit. This effectively makes all zeroes coincide in the $z^{\prime}$ plane while keeping the poles finite. Note that $k$ is not being scaled with $\epsilon$.

As mentioned in section 2., we use the equations (2.9), (2.14) and (2.15) to write the cross ratio $\eta$ on the world sheet in terms of the perimeters $r_{i}$ and the Strebel 
lengths $a, b$. We now combine this with a systematic expansion scheme in $\epsilon$. The strategy of the approximation scheme is to first perform a Taylor series expansion of (2.9), (2.14), (2.15) about the specific point $\bar{u}$ in the $u$-plane, which corresponds to large $z$. Then we can solve for $\eta$ order by order in a series expansion in $\epsilon$.

Solving for $u$ in terms of $z$, using the change of variables in (2.4), we can set up the following asymptotic expansion

$$
\begin{aligned}
u & =\int_{1}^{\infty} \frac{d z}{w}-\int_{\frac{z^{\prime}}{\epsilon}}^{\infty} \frac{d z}{w} \\
& =\bar{u}-\frac{\epsilon}{k z^{\prime}}-\frac{1}{6 k}\left(1+\frac{1}{k^{2}}\right) \frac{\epsilon^{3}}{z^{\prime 3}}+\cdots
\end{aligned}
$$

Here we have expanded the integrand in $\epsilon$ and integrated term by term, showing terms upto $O\left(\epsilon^{3}\right)$ for future purposes. In (3.1) $\bar{u}$ is given by

$$
\begin{aligned}
\bar{u} & =\int_{1}^{\infty} \frac{d z}{\sqrt{\left(z^{2}-1\right)\left(z^{2} k^{2}-1\right)}} \\
& =\frac{\omega_{1}}{2}+\omega_{2} .
\end{aligned}
$$

The easiest way to fix $\bar{u}$ is to look at the equation for $z$ in terms of $\bar{u}$ for large $z$. From (3.1) this is given by

$$
z \sim-\frac{1}{k(u-\bar{u})} .
$$

This indicates that $\bar{u}$ is a pole with residue $-\frac{1}{k}$. The fact that $z=\operatorname{sn}\left(u+\omega_{1} / 2\right)$, and that $\operatorname{sn}(u)$ has a pole with residue $-1 / k$ at $\omega_{1}+\omega_{2}$ (see table 1 ) fixes $\bar{u}=\omega_{1} / 2+\omega_{2}$.

To perform the large $z$ expansion in (2.9) we need to write $\operatorname{sn}\left(u_{i}\right), \operatorname{cn}\left(u_{i}\right), \operatorname{dn}\left(u_{i}\right)$ in terms of the asymptotic expansion in $1 / z_{i}$ around the point $\bar{u}$. This is done in appendix D, and from (D.3), we can read off the leading terms which are relevant for us in this section.

$$
\sum_{i=0}^{3} r_{i}=0, \quad \sum_{i=0}^{3} r_{i} x_{i}=0, \quad \sum_{i=0}^{3} r_{i} x_{i}^{2}=0 .
$$

Here $x_{i} \equiv \frac{1}{z_{i}^{\prime}}$ are finite as $\epsilon \rightarrow 0$. As can be seen from the scaling of $\phi(z)$ with $\epsilon$ or as we will see explicitly in Sec.5, the equations (2.14) and (2.15) for the Strebel lengths $a$ and $b$ can also be expanded in powers of $\epsilon$. The leading nonzero terms are of $O\left(\epsilon^{3}\right)$. So for the leading order solution of the Strebel conditions, we will not need these equations. 
Therefore we have only the leading order equations in (3.4) to solve. In terms of $x_{i}=y_{i}+x_{0}$, we can easily verify that (3.4) simplifies to

$$
\begin{aligned}
r_{0}+r_{1}+r_{2}+r_{3} & =0, \\
r_{1} y_{1}+r_{2} y_{2}+r_{3} y_{3} & =0, \\
r_{1} y_{1}^{2}+r_{2} y_{2}^{2}+r_{3} y_{3}^{2} & =0 .
\end{aligned}
$$

In other words, the translational mode $x_{0}$ drops out of these equations. Note that the first equation is a constraint among the perimeters (residues) of the Strebel differential. The object of interest to us, the cross ratio of the poles

$$
\begin{aligned}
\eta & =\frac{\left(z_{3}-z_{2}\right)\left(z_{1}-z_{0}\right)}{\left(z_{1}-z_{2}\right)\left(z_{3}-z_{0}\right)} \\
& =\frac{y_{1}\left(y_{3}-y_{2}\right)}{y_{3}\left(y_{1}-y_{2}\right)} .
\end{aligned}
$$

depends only the ratio of the $y$ 's (and is also independent of $x_{0}$ ). In fact, defining

$$
w_{1}=\frac{y_{1}}{y_{3}}, \quad w_{2}=\frac{y_{2}}{y_{3}}
$$

the cross ratio (3.6) is simply

$$
\eta=w_{1} \frac{1-w_{2}}{w_{1}-w_{2}},
$$

We can solve for $w_{1}, w_{2}$ using the last two equations of (3.5). Eliminating $w_{2}$ in favour of $w_{1}$ using the second equation in (3.5) and substituting it into the third equation yields

$$
r_{1}\left(r_{1}+r_{2}\right) w_{1}^{2}+2 r_{1} r_{3} w_{1}+r_{3}\left(r_{3}+r_{2}\right)=0
$$

We have

$$
w_{1}=\frac{y_{1}}{y_{3}}=\frac{-r_{1} r_{3} \pm \sqrt{r_{1} r_{2} r_{3} r_{0}}}{r_{1}\left(r_{1}+r_{2}\right)}
$$

and as a result

$$
w_{2}=\frac{y_{2}}{y_{3}}=\frac{-r_{2} r_{3} \mp \sqrt{r_{1} r_{2} r_{3} r_{0}}}{r_{2}\left(r_{1}+r_{2}\right)} .
$$

To connect with the positive Strebel lengths, we will take $r_{0}=-p_{0}<0$ and $r_{i}=p_{i}$ for $(i=1,2,3)$. This reflects the fact that $p_{0}=p_{1}+p_{2}+p_{3}$ is the relation among the perimeters for the dual graph to the $Y$ diagram. 
Then, on substituting the values for $w_{1}$ and $w_{2}$ into (3.8) we obtain, after some simplifications

$$
\eta=\left(\frac{\sqrt{p_{0} p_{2}} \pm i \sqrt{p_{1} p_{3}}}{p_{1}+p_{2}}\right)^{2}
$$

Note that this zeroth order solution did not require knowledge of the modulus $k$. In appendix C. we obtain this result in an elementary fashion and show how it is equivalent to the solution obtained in [43]. Without loss of generality, we can take the plus sign in (3.12), since the other choice is just the complex conjugate.

\section{A worldsheet four point function}

What we saw in the previous section is that for a $Y$ shaped skeleton Feynman diagram the Strebel differential is particularly simple. Aharony et.al. 443 exploited this property to study the contribution from the $Y$ diagram to the correlator $\left\langle\operatorname{Tr} \Phi^{J_{1}}\left(x_{1}\right) \operatorname{Tr} \Phi^{J_{2}}\left(x_{2}\right) \operatorname{Tr} \Phi^{J_{3}}\left(x_{3}\right) \operatorname{Tr} \Phi^{J}(0)\right\rangle$ in free field theory $\left(J=J_{1}+J_{2}+J_{3}\right)$. As mentioned in the introduction, here we will instead study the correlator

$$
\Gamma_{\left\{J_{i}\right\}}^{(4)}\left(x_{1}, x_{2}, x_{3}\right)=\left\langle\operatorname{Tr} X^{J_{1}}\left(x_{1}\right) \operatorname{Tr} Y^{J_{2}}\left(x_{2}\right) \operatorname{Tr} Z^{J_{3}}\left(x_{3}\right) \operatorname{Tr}\left(\bar{X}^{J_{1}} \bar{Y}^{J_{2}} \bar{Z}^{J_{3}}\right)(0)\right\rangle,
$$

since it gets contributions only from the $Y$ diagram (see footnote 4 ) in the free theory. The answer in the free field theory is, of course, simply

$$
\left\langle\operatorname{Tr} X^{J_{1}}\left(x_{1}\right) \operatorname{Tr} Y^{J_{2}}\left(x_{2}\right) \operatorname{Tr} Z^{J_{3}}\left(x_{3}\right) \operatorname{Tr}\left(\bar{X}^{J_{1}} \bar{Y}^{J_{2}} \bar{Z}^{J_{3}}\right)(0)\right\rangle=\frac{\tilde{C}\left(J_{i}\right)}{x_{1}^{2 J_{1}} x_{2}^{2 J_{2}} x_{3}^{2 J_{3}}} .
$$

In the position space Schwinger representation, we can write this as

$$
\begin{aligned}
& \left\langle\operatorname{Tr} X^{J_{1}}\left(x_{1}\right) \operatorname{Tr} Y^{J_{2}}\left(x_{2}\right) \operatorname{Tr} Z^{J_{3}}\left(x_{3}\right) \operatorname{Tr}\left(\bar{X}^{J_{1}} \bar{Y}^{J_{2}} \bar{Z}^{J_{3}}\right)(0)\right\rangle \\
= & C\left(J_{i}\right) \int_{0}^{\infty} d \sigma_{1} d \sigma_{2} d \sigma_{3} \sigma_{1}^{J_{1}-1} \sigma_{2}^{J_{2}-1} \sigma_{3}^{J_{3}-1} e^{-\left(\sigma_{1} x_{1}^{2}+\sigma_{2} x_{2}^{2}+\sigma_{3} x_{3}^{2}\right)}
\end{aligned}
$$

We now need to change variables from the three $\sigma_{i}$ to $\eta$ and an overall scaling factor, to be able to write (4.2) as a closed string integral. The three $\sigma_{i}$ are identified as per 37] with the three independent Strebel lengths corresponding to the dual graph. They are, in fact, the three independent residues at the poles 0,1 and $\infty$ of the Strebel differential in (2.3).

$$
\sigma_{i}=p_{i}, \quad(i=1,2,3)
$$


We saw that the complex cross ratio $\eta$ is given by (3.12) to be

$$
\eta=\left(\frac{\sqrt{p_{0} p_{2}}+i \sqrt{p_{1} p_{3}}}{p_{1}+p_{2}}\right)^{2},
$$

with $p_{0}=p_{1}+p_{2}+p_{3}$. Notice that $\eta$ actually depends only on the two independent ratios $s_{1}=\frac{p_{1}}{p_{3}}=\frac{\sigma_{1}}{\sigma_{3}}$ and $s_{2}=\frac{p_{2}}{p_{3}}=\frac{\sigma_{2}}{\sigma_{3}}$

$$
\eta=\left(\frac{\sqrt{s_{0} s_{2}}+i \sqrt{s_{1}}}{s_{1}+s_{2}}\right)^{2}
$$

with $s_{0}=\frac{p_{0}}{p_{3}}=1+s_{1}+s_{2}$.

We can solve (4.6) for $s_{1}$ and $s_{2}$ in terms of $\eta$. Firstly, it is easy to check that with $\eta=|\eta| e^{i \theta}$, we have

$$
|\eta|=\frac{1+s_{2}}{s_{1}+s_{2}}, \quad 1-\cos \theta=\frac{2 s_{1}}{\left(1+s_{2}\right)\left(s_{1}+s_{2}\right)} .
$$

Therefore

$$
|1-\eta|=\frac{1+s_{1}}{s_{1}+s_{2}}
$$

so that one has

$$
s_{1}=\frac{(1-|\eta|+|1-\eta|)}{(-1+|\eta|+|1-\eta|)}
$$

and

$$
s_{2}=\frac{(1+|\eta|-|1-\eta|)}{(-1+|\eta|+|1-\eta|)} .
$$

Finally,

$$
s_{0}=1+s_{1}+s_{2}=\frac{(1+|\eta|+|1-\eta|)}{(-1+|\eta|+|1-\eta|)} .
$$

Coming back to (4.3), we can write it as

$$
\begin{aligned}
\Gamma_{\left\{J_{i}\right\}}^{(4)}\left(x_{i}\right) & =C\left(J_{i}\right) \int_{0}^{\infty} d \sigma_{3} \sigma_{3}^{J-1} \int d s_{1} d s_{2} s_{1}^{J_{1}-1} s_{2}^{J_{2}-1} e^{-\sigma_{3}\left(s_{1} x_{1}^{2}+s_{2} x_{2}^{2}+x_{3}^{2}\right)} \\
& =(J-1) ! C\left(J_{i}\right) \int d s_{1} d s_{2} \frac{s_{1}^{J_{1}-1} s_{2}^{J_{2}-1}}{\left(s_{1} x_{1}^{2}+s_{2} x_{2}^{2}+x_{3}^{2}\right)^{J}}
\end{aligned}
$$

Note that $J=J_{1}+J_{2}+J_{3}$. The measure term

$$
d s_{1} d s_{2}=J(\eta, \bar{\eta}) d^{2} \eta
$$

where the Jacobian can be explicitly worked out, using (4.9) (4.10), to be

$$
J(\eta, \bar{\eta})=\frac{1}{D^{3}} \frac{|\eta-\bar{\eta}|}{|\eta||1-\eta|},
$$


with $D=(-1+|\eta|+|1-\eta|)$. Using the identity

$$
|\eta-\bar{\eta}|=[(1+|\eta|+|1-\eta|)(1-|\eta|+|1-\eta|)(1+|\eta|-|1-\eta|)(-1+|\eta|+|1-\eta|)]^{\frac{1}{2}}
$$

and equations (4.9), 4.10) we can finally rewrite (4.12) as

$$
\begin{array}{r}
\Gamma_{\left\{J_{i}\right\}}^{(4)}\left(x_{i}\right) \equiv \int d^{2} \eta G_{\left\{x_{i}\right\}}^{\left\{J_{i}\right\}}(\eta, \bar{\eta})=\int d^{2} \eta \frac{(1+|\eta|+|1-\eta|)^{\frac{1}{2}}}{|\eta||1-\eta|} \times \\
\frac{(1-|\eta|+|1-\eta|)^{J_{1}-\frac{1}{2}}(1+|\eta|-|1-\eta|)^{J_{2}-\frac{1}{2}}(-1+|\eta|+|1-\eta|)^{J_{3}-\frac{1}{2}}}{\left[x_{1}^{2}(1-|\eta|+|1-\eta|)+x_{2}^{2}(1+|\eta|-|1-\eta|)+x_{3}^{2}(-1+|\eta|+|1-\eta|)\right]^{J}}
\end{array}
$$

We thus obtain the expression (1.5) for the integrand $G_{\left\{x_{i}\right\}}^{\left\{J_{i}\right\}}(\eta, \bar{\eta})$ on the moduli space of the four punctured sphere.

As mentioned in the introduction, for $G_{\left\{x_{i}\right\}}^{\left\{J_{i}\right\}}(\eta, \bar{\eta})$ to be interpretable as a worldsheet four point function of physical vertex operators (1.6), a number of requirements on its form have to be satisfied. The simplest is that of crossing symmetry.

$$
\begin{aligned}
G_{x_{2} x_{1} x_{3}}^{J_{2} J_{1} J_{3} J}(1-\eta, 1-\bar{\eta}) & =G_{x_{1} x_{2} x_{3}}^{J_{1} J_{2} J_{3} J}(\eta, \bar{\eta}), \\
G_{x_{3} x_{2} x_{1}}^{J_{3} J_{2} J_{1} J}\left(\frac{1}{\eta}, \frac{1}{\bar{\eta}}\right) & =|\eta|^{4} G_{x_{1} x_{2} x_{3}}^{J_{1} J_{2} J_{3} J}(\eta, \bar{\eta}) .
\end{aligned}
$$

The weight $|\eta|^{4}$ in the second line is the same as the requirement that $\mathcal{V}^{J}$ in (1.6) is a $(1,1)$ vertex operator on the worldsheet. Note that the crossing symmetry requirement is not just a consequence of $S L(2, C)$ invariance, but also of the fact that there is an additional permutation symmetry among the labels when we consider a correlator of primary operators. This is reflected in the fact that the functional form of the correlator $G_{\left\{x_{i}\right\}}^{\left\{J_{i}\right\}}$ is unchanged in Eqs. (4.17).

It is a nice feature of the change of variables we have used that crossing symmetry is built in. We can indeed check that the change of variables in equations (4.9) (4.10) implements the permutation symmetry between the labels $(1,2,3)$ in the way we expect $^{6}$. Therefore it is assured that the $G_{\left\{x_{i}\right\}}^{\left\{J_{i}\right\}}(\eta, \bar{\eta})$ will satisfy the requirements of (4.17). This is, in fact, easy to verify explicitly from the expression (1.5).

Another requirement for a CFT correlator in a string theory is having an OPE consistent with locality. In other words, in the expansion (1.8) in powers $\eta^{h} \bar{\eta}^{\bar{h}}$, the correlator must have $(h-\bar{h})$ always an integer. In 43] the form of the correlator

\footnotetext{
${ }^{6}$ We thank A. Sen for useful discussions on this point.
} 
was given to the first couple of orders in a somewhat different variable $t$ (which is to leading order the same as $\eta$ ) and it was found that the powers obeyed this property, though $h, \bar{h}$ could be individually half integers. From the explicit expression in (4.16), this is actually manifest to all orders in the expansion for the $G_{\left\{x_{i}\right\}}^{\left\{J_{i}\right\}}(\eta, \bar{\eta})$.

Finally, we would like to be able to see if $G_{\left\{x_{i}\right\}}^{\left\{J_{i}\right\}}(\eta, \bar{\eta})$ actually arises as the correlator of local vertex operators. The fairly simple dependence of $G_{\left\{x_{i}\right\}}^{\left\{J_{i}\right\}}(\eta, \bar{\eta})$ on $|\eta|$ and $|1-\eta|$ are indicative of local dependence on the points $0,1, \infty$ and $\eta$. Indeed, as was pointed out to us by D. Gaiotto, the expressions that enter here are very closely related to similar ones in the four point functions of spin fields in the Ising model.

For instance, the correlator of four order operators $\sigma(z, \bar{z})$ (see equation (12.63) of 49]) is given by

$$
\langle\sigma(1) \sigma(2) \sigma(3) \sigma(4)\rangle=\sqrt{\frac{1}{2\left|z_{13} z_{14}\right|^{\frac{1}{2}}}} \frac{1}{\sqrt{|\eta||1-\eta|}}(1+|\eta|+|1-\eta|)^{\frac{1}{2}} .
$$

and that of two order and two disorder operators ( equation (12.66) in 49]) is

$$
\langle\sigma(1) \mu(2) \sigma(3) \mu(4)\rangle=\sqrt{\frac{1}{2\left|z_{13} z_{14}\right|^{\frac{1}{2}}}} \frac{1}{\sqrt{|\eta||1-\eta|}}(-1+|\eta|+|1-\eta|)^{\frac{1}{2}} .
$$

By permuting the ordering of the operators in (4.19), we get the last factor proportional in turn to $(1-|\eta|+|1-\eta|)^{\frac{1}{2}}$ and $(1+|\eta|-|1-\eta|)^{\frac{1}{2}}$. These are exactly the ingredients that enter into the correlator defined by (4.16). Thus it is not far fetched to imagine that the entire expression in (4.16) might arise as a local correlator in a CFT. Since the spin field correlators seem like building blocks for the actual object that appears here, It would be very interesting to use them to try and reconstruct the worldsheet CFT, at least in an algebraic way. For instance, knowing the conformal blocks of the Ising model, we can try and decompose our candidate correlator into candidate conformal blocks.

Another potentially interesting connection suggested by the appearance of Ising model correlation functions is to $W$-strings (see [50] [51] [52] for reviews). Ising model correlation function are known to appear in the four point scattering amplitudes of $W_{3}$ strings $[53]^{7}{ }^{8}$. This maybe a natural connection given the fact that the free

\footnotetext{
${ }^{7}$ We thank Ari Pakman for bringing this reference to our attention.

${ }^{8}$ The Ising model has also arisen in $\operatorname{Spin}(7)$ compactifications 54 .
} 
gauge theory has higher spin symmetries in spacetime which might well be reflected as higher spin symmetries on the worldsheet in the usual manner by which spacetime and worldsheet symmetries are related.

In any case, we believe there is enough reason to take $G_{\left\{x_{i}\right\}}^{\left\{J_{i}\right\}}(\eta, \bar{\eta})$ seriously as a candidate four point function in the as yet unknown worldsheet theory of $A d S_{5} \times S^{5}$ dual to free $\mathcal{N}=4$ Yang-Mills theory.

\section{A perturbation scheme around the $Y$ diagram}

In this section we develop a systematic approximation scheme around the Y diagram. We use the equations (2.9), (2.14) and (2.15) to write the cross ratio on the world sheet in terms of the perimeters $r_{i}$ and the Strebel lengths $a, b$. As we have seen in Section 3, the Y diagram corresponds to the large $z$ limit of the general Strebel differential given in (2.3). In the $u$-plane, this point corresponds to $\bar{u}=\omega_{1} / 2+\omega_{2}$. In Section 3. we have already used the leading terms in the expansion to obtain the solution shown in (3.12). We now retain the first two leading terms in the equations (D.3) and the Strebel length equations (2.14), (2.15) to obtain the leading correction in $\epsilon$ to the cross-ratio. From (D.3) we see that the three conditions on the poles $(2.9)$ in this approximation reduce to

$$
\begin{aligned}
\sum_{i=0}^{3} r_{i}-\epsilon^{4} \tilde{E} \sum_{i=0}^{3} r_{i} x_{i}^{4} & =0, \\
\sum_{i=0}^{3} r_{i} x_{i}-\epsilon^{2} E \sum_{i=0}^{3} r_{i} x_{i}^{3} & =0, \\
\sum_{i=0}^{3} r_{i} x_{i}^{2}-\epsilon^{2} E \sum_{i=0}^{3} r_{i} x_{i}^{4} & =0 .
\end{aligned}
$$

where

$$
\tilde{E}=-\frac{\left(1-k^{2}\right)^{2}}{8 k^{4}}, \quad E=-\frac{1}{2}\left(1+\frac{1}{k^{2}}\right)
$$

It is clear from the expansions that we have retained terms only to $O\left(\epsilon^{4}\right)$.

The next set of equations to expand are (2.14) and (2.15). We expand the variables $u_{i}$ occurring in these equations about $\bar{u}$ in terms of $x_{i}$ and performing simple algebraic manipulations (see appendix D. for details) we obtain the following 
equivalent equations valid up to $O\left(\epsilon^{4}\right)$

$$
\begin{aligned}
a & =\epsilon^{3} p_{1} \sum r_{i} x_{i}^{3}+\epsilon^{4} p_{2} \sum r_{i} x_{i}^{4}, \\
a \omega_{2}+b \omega_{1} & =\epsilon^{3} q_{1} \sum r_{i} x_{i}^{3}+\epsilon^{4} q_{2} \sum r_{i} x_{i}^{4},
\end{aligned}
$$

where $p_{1}, q_{1}, p_{2}, q_{2}$ are constants depending on the value of $\bar{u}$, as given in (D.6), (D.8) (D.10). For our purpose here, it is sufficient to provide the following information about the constants which are valid to the $O\left(\epsilon^{0}\right)$

$$
\begin{aligned}
\frac{p_{1}^{(0)}}{q_{1}^{(0)}} & =2 i\left(\frac{1-10 k^{2}+k^{4}}{12} \vartheta_{3}^{2}(q)+\frac{1}{12} \frac{1}{\vartheta_{3}^{2}(q)} \frac{\vartheta_{1}^{\prime \prime \prime}(q)}{\vartheta_{1}^{\prime}(q)}\right), \\
q_{1}^{(0)} & =-\frac{\pi}{3 k}\left(1+\frac{1}{k^{2}}\right), \\
\omega_{1} & =\pi \vartheta_{3}^{2}(q), \quad q=\exp \left(2 \pi i \frac{\omega_{1}}{\omega_{2}}\right) .
\end{aligned}
$$

The periods $\left(2 \omega_{1}, 2 \omega_{2}\right)$ of the auxilliary torus in the $u$ plane are functions of the modulus $k$, they are all $O\left(\epsilon^{0}\right)$. In the above equation the superscript ${ }^{(0)}$ refer to the order in $\epsilon$. It is clear from (5.3) that the Strebel lengths $a$ and $b$ begin at $O\left(\epsilon^{3}\right)$. This fact can also be seen easily by performing the scaling $z=z^{\prime} / \epsilon$ in the equation for the Strebel differential (2.3). We can therefore expand the Strebel lengths as

$$
a=a^{(3)} \epsilon^{3}+a^{(4)} \epsilon^{4}+\ldots, \quad b=b^{(3)} \epsilon^{3}+b^{(4)} \epsilon^{4}+\ldots
$$

For convenience we define

$$
c=\sum r_{i} x_{i}^{3}, \quad \tilde{c}=\sum r_{i} x_{i}^{4}
$$

From (5.3) it is clear that $c, c^{\prime}$ begins at $O\left(\epsilon^{0}\right)$. Now comparing terms of order $O\left(\epsilon^{3}\right)$ in the first equation of (5.3) we obtain the following relation

$$
c^{(0)}=\sum r_{i} x_{i}^{(0) 3}=\frac{a^{(3)}}{p_{1}^{(0)}} .
$$

Furthermore, comparing the terms of $O\left(\epsilon^{3}\right)$ in both the equations and eliminating $c$ from them we obtain

$$
\frac{p_{1}^{(0)}}{q_{1}^{(0)}}\left(a^{(3)} \omega_{2}^{(0)}+b^{(3)} \omega_{1}^{(0)}\right)=a^{(3)} .
$$

It is this equation which determines $k$ in terms of $a^{(3)}$ and $b^{(3)}$. One substitutes the values of $\omega_{1}$ and $\omega_{2}$ in terms of $k$, uses (5.4) and then solves for $k$ using the above 
equation. This is done in appendix E. From now on, we assume that $k=k(a, b)$, and similarly $\omega_{1}=\omega_{1}(a, b)$ and $\omega_{2}=\omega_{2}(a, b)$ since each of them are functions of $k$. Note that $k$ starts at $O\left(\epsilon^{0}\right)$.

Now that we have the basic equations (5.1), (5.3) and (5.8) we can organize them so that one can solve for the $x_{i}$ using perturbation theory. To isolate the translational degree of freedom in the variables $x_{i}$ we first write $x_{1}=y_{1}+x_{0}, x_{2}=y_{2}+x_{0}, x_{3}=$ $y_{3}+x_{0}$. Therefore the variables to solve for are now $y_{1}, y_{2}, y_{3}, x_{0}$. Then the equations (5.1) and (5.6) reduce to

$$
\begin{aligned}
\sum_{i=1}^{3} r_{i} & =\epsilon^{4} \tilde{E} \tilde{c}, \\
\sum_{i=1}^{3} r_{i} y_{i}+\epsilon^{4} \tilde{E} \tilde{c} x_{0} & =\epsilon^{2} E c \\
\sum_{i=1}^{3} r_{i} y_{i}^{2}+2 \epsilon^{2} E c x_{0}-\epsilon^{4} \tilde{E} \tilde{c} x_{0}^{2} & =\epsilon^{2} E \tilde{c}, \\
\sum_{i=1}^{3} r_{i} y_{i}^{3}+3 \epsilon^{2} E \tilde{c} x_{0}-3 \epsilon^{2} E c x_{0}^{2}+\epsilon^{4} \tilde{E} \tilde{c} x_{0}^{3} & =c \\
\sum_{i=1}^{3} r_{i} y_{i}^{4}+4 c x_{0}-6 \epsilon^{2} E \tilde{c} x_{0}^{2}+4 \epsilon^{2} E c x_{0}^{3}-\epsilon^{4} \tilde{E} \tilde{c} x_{0}^{4} & =\tilde{c} .
\end{aligned}
$$

To obtain these equations we have repeatedly used the basic equations (5.1) and (5.6). Note that the first equation in (5.9) determines the constant $\tilde{c}$ since the perimeters $r_{i}$ are given to us. The first equation indicates the constraint $\sum_{i=0}^{3} r_{i}=0$ holds till $O\left(\epsilon^{4}\right)$. The constant $c$ is determined from (5.7) and finally the constants $E$ and $\tilde{E}$ are defined in (5.2). As mentioned earlier, the modulus $k$ is determined as a function of $a, b$ from (5.8). To summarize, (5.9) can be solved using perturbation theory for the variables $y_{1}, y_{2}, y_{3}, x_{0}$ in terms of the perimeters $r_{i}$ and the Strebel lengths $a, b$. We can obtain the cross ratio from the equation (3.8).

We now set about to solve the above equations and obtaining the leading correction. From the first two equations in (5.9) we see that the next non-trivial correction to the cross ratio occurs at $O\left(\epsilon^{2}\right)$. These equations determine the ratios $w_{1}=y_{1} / y_{3}, w_{2}=y_{2} / y_{3}$ to $O\left(\epsilon^{2}\right)$

$$
r_{1} w_{1}+r_{2} w_{2}+r_{3}=\epsilon^{2} \frac{E c^{(0)}}{y_{3}^{(0)}}
$$




$$
r_{1} w_{1}^{2}+r_{2} w_{2}^{2}+r_{3}+2 \epsilon^{2} E c^{(0)} \frac{x_{0}^{(0)}}{y_{3}^{(0) 2}}=\epsilon^{2} \frac{E \tilde{c}^{(0)}}{y_{3}^{(0) 2}} .
$$

These equations indicate that we need to need the information of $y_{3}$ and $x_{0}$ at the zeroth order. This is obtained from using the last two equations of (5.9) at the zeroth order, these are

$$
\sum_{i=1}^{3} r_{i} y_{i}^{3}=c^{(0)}, \quad \sum_{i=1}^{3} r_{i} y_{i}^{4}+4 c^{(0)} x_{0}=\tilde{c}^{(0)}
$$

Substituting the zeroth order values of $y_{1}, y_{2}$ in terms of $r_{i}$ and $y_{3}$ in the above equation we obtain

$$
y_{3}^{(0)}=\left(\frac{c^{(0)} r_{1} r_{2}\left(r_{1}+r_{2}\right)^{2}}{r_{3}\left\{r_{1} r_{2} r_{3}\left(r_{0}-r_{3}\right) \pm\left(r_{2}-r_{1}\right) \sqrt{r_{1} r_{2} r_{3} r_{0}}\right\}}\right)^{1 / 3}
$$

where $c^{(0)}$ is obtained from (5.7). Similarly from the second equation in (5.11) we can obtain $x_{0}$ as

$$
\begin{aligned}
x_{0}^{(0)}= & \frac{\tilde{c}^{(0)}}{4 c^{(0)}}-\frac{\left(y_{3}^{(0}\right)^{4}}{4 c^{(0)}}\left(\frac{r_{3} r_{0}}{r_{1} r_{2}\left(r_{1}+r_{2}\right)^{3}}\right) \times \\
& \left\{r_{1} r_{2}\left(r_{0} r_{3}-r_{0}^{2}-4 r_{3}^{2}\right)+r_{3} r_{0}\left(r_{1}+r_{2}\right)^{2} \pm 4 r_{3}\left(r_{2}-r_{1}\right) \sqrt{r_{1} r_{2} r_{3} r_{0}}\right\} .
\end{aligned}
$$

Here one has to substitute $y_{3}$ from (5.12), $c$ from (5.7) and $\tilde{c}$ from the first line of (5.9)

$$
\tilde{c}^{(0)}=\sum r_{i}^{(4)} / \tilde{E}
$$

Now that we have all the quantities entering into (5.10), we can solve for $w_{1}$ and $w_{2}$. For convenience we rewrite (5.10) as

$$
\begin{aligned}
& r_{1} w_{1}+r_{2} w_{2}=-r_{3}-\epsilon^{2} \delta_{1}, \\
& r_{1} w_{1}^{2}+r_{2} w_{2}^{2}=-r_{3}-\epsilon^{2} \delta_{2},
\end{aligned}
$$

where

$$
\delta_{1}=-\frac{E c^{(0)}}{y_{3}^{(0)}}, \quad \delta_{2}=-\frac{E}{y_{3}^{(0) 2}}\left(\tilde{c}^{(0)}-2 c^{(0)} x_{0}^{(0)}\right) .
$$

Eliminating $w_{2}$ using the first equation of (5.15) in the second equation we obtain the following quadriatic equation where we have retained terms to $O\left(\epsilon^{2}\right)$.

$$
r_{1}\left(r_{1}+r_{2}\right) w_{1}^{2}+\left(2 r_{1} r_{3}+2 \epsilon^{2} r_{1} \delta_{1}\right) w_{1}+r_{3}\left(r_{3}+r_{2}\right)+r_{2} \epsilon^{2} \delta_{2}+2 r_{3} \epsilon^{2} \delta_{1}=0
$$


The solution for $w_{1}$ and $w_{1}$ are given by

$$
\begin{aligned}
w_{1} & =-\frac{1}{r_{1}\left(r_{1}+r_{2}\right)}\left[r_{1} r_{3} \pm \sqrt{r_{1} r_{2} r_{3} r_{0}}\right. \\
& \left.+\epsilon^{2}\left(r_{1} \delta_{1} \pm \sqrt{r_{1} r_{2} r_{3} r_{0}}\left(-\frac{\delta_{1}}{r_{0}}+\left(\frac{1}{r_{3}}+\frac{1}{r_{0}}\right) \frac{\delta_{2}}{2}\right)\right)\right], \\
w_{2} & =-\frac{1}{r_{2}\left(r_{1}+r_{2}\right)}\left[r_{2} r_{3} \mp \sqrt{r_{1} r_{2} r_{3} r_{0}}\right. \\
& \left.+\epsilon^{2}\left(r_{2} \delta_{1} \mp \sqrt{r_{1} r_{2} r_{3} r_{0}}\left(-\frac{\delta_{1}}{r_{0}}+\left(\frac{1}{r_{3}}+\frac{1}{r_{0}}\right) \frac{\delta_{2}}{2}\right)\right)\right] .
\end{aligned}
$$

From this we can evaluate the cross-ratio using (3.8), which is given by

$$
\begin{aligned}
\eta_{ \pm} & =\eta_{ \pm}^{(0)}+\epsilon^{2} \frac{\sqrt{p_{2} p_{0}} \pm i \sqrt{p_{1} p_{3}}}{\left(p_{1}+p_{2}\right)^{2}}\left(\delta_{1}\left(\sqrt{\frac{p_{2}}{p_{0}}} \pm i \sqrt{\frac{p_{1}}{p_{3}}}\right)+\kappa\left(\sqrt{p_{2} p_{0}} \pm i \sqrt{p_{1} p_{3}}\right)\right) \\
& -\epsilon^{2} \kappa \eta_{ \pm}^{(0)}, \\
\text { where } \kappa & =-\frac{\delta_{1}}{r_{0}}+\left(\frac{1}{r_{3}}+\frac{1}{r_{0}}\right) \frac{\delta_{2}}{2} .
\end{aligned}
$$

Here $\eta_{ \pm}^{(0)}$ is the zeroth order solution for the cross ratio given in (3.12).

\section{Conclusions}

We have seen that we can arrive at explicit expressions for candidate worldsheet correlators, in the string dual to free Yang-Mills theory by using a definite change of variables on the Schwinger representation of field theory amplitudes. These candidates seem quite promising in that they obey many of the properties one might expect of them. Moreover, their form seems to suggest ways of potentially gaining a better understanding of the worldsheet theory. The relation with the spin field correlators might be a fruitful way to both uncover the structure of conformal blocks and maybe even give some clues about the worldsheet action. One point that needs better understanding here is the fact that the spacetime special conformal transformations do not act locally in this Schwinger representation [13]. Perhaps for the cases of spacetime conformal invariance, we can modify the prescription to make this symmetry manifest on the worldsheet as well.

It will also be important to have at hand a large number of explicitly worked out correlators. The case of extremal correlators is particularly interesting, affording a way to potentially compare with large radius intuition as well as methods. There are also other special four point diagrams which, as observed in [43], seem to get 
contributions only from real subspaces of moduli space. It would be good to get a better grasp of what is going on here by comparing with cases such as the $Y$ diagram which yield local expressions on the worldsheet.

To summarise, we are hopeful that this approach to obtaining the worldsheet theory will be fruitful, like in the Liouville case, in effectively bypassing an action formulation of the theory and yet capturing all the essential information.

\section{Acknowledgments}

We would like to thank A. Dhar, E. Gava, S. Giddings, D. Ghoshal, D. Gross, L. Motl, K.S. Narain, A. Pakman, J. Polchinski, A. Strominger and C. Vafa for useful discussions. Special thanks are due to D. Gaiotto, S. Minwalla and A. Sen for insightful remarks during the course of this work. It is also a pleasure to thank O. Aharony, Z. Komargodski and S. Razamat for discussions and specially for their very useful comments on a preliminary draft of this paper. J.R.D. wishes to thank the Weizmann Institute, ASICTP and CERN for hospitality during the course of this work. R. G. would like to thank the string theory group at Harvard University, the organisers of the "New Topological Structures in Physics" workshop at MSRI, Berkeley, the Physics Department, UC Santa Barbara and TIFR, Mumbai for hospitality while this work was being completed. We would also jointly like to thank the organisers of the Indo-Israeli workshop on String Theory at Ein Boqeq for their hospitality. Finally, we are indebted to the people of India for their unstinting support for our discipline.

\section{A. Elliptic Functions}

In this section we briefly review the properties of elliptic functions which are used extensively in this paper. A detailed discussion is available in [55]

\section{The Jacobian elliptic functions}

The elliptic function sn $u$ is defined as follows, consider the integral

$$
u=\int_{0}^{z} d t\left(1-t^{2}\right)^{-\frac{1}{2}}\left(1-k^{2} t^{2}\right)^{-\frac{1}{2}},
$$


then $z=\operatorname{sn}(u, k)$, we will supress the modulus $k$ unless different. The complementary modulus $k^{\prime}$ is defined by $k^{2}+k^{\prime 2}=1$ The elliptic functions $\operatorname{cn}(u)$ and $\operatorname{dn}(u)$ are defined from

$$
\operatorname{sn}^{2} u+\operatorname{cn}^{2} u=1, \quad k^{2} \operatorname{sn}^{2} u+\operatorname{dn}^{2} u=1 .
$$

The periods $K$ and $i K^{\prime}$ of the elliptic functions are defined by the integrals

$$
K=\int_{0}^{1} d t\left(1-t^{2}\right)^{-\frac{1}{2}}\left(1-k^{2} t^{2}\right)^{-\frac{1}{2}},
$$

where the integral is along the straight line from 0 to $1 . K^{\prime}$ is given by

$$
K^{\prime}=\int_{1}^{1 / k} d t\left(t^{2}-1\right)^{-\frac{1}{2}}\left(1-k^{2} t^{2}\right)^{-\frac{1}{2}}
$$

here the branch cuts are chosen from 0 to $-\infty$ and 1 to $\infty$. We define the periods $\omega_{1}$ and $\omega_{2}$ by

$$
\omega_{1}=2 K, \quad \omega_{2}=i K^{\prime}
$$

Note that $K, K^{\prime}$ and therefore $\omega_{1}, \omega_{2}$ are functions of the modulus $k$ through the integrals (A.3) and (A.4). From (A.1) it is easy to see that $\operatorname{sn} u$ is an odd function of $u$. The elliptic functions $c n u$ and $\operatorname{dn} u$ are even functions of $u$. In the table below we summarize the information about the periods, the poles and the respective residues and the zeros of the corresponding elliptic functions.

\begin{tabular}{l|l|l|l|l} 
Function & Periods & Poles & Residues & Zeros \\
\hline $\operatorname{sn} u$ & $\left(2 \omega_{1}, 2 \omega_{2}\right)$ & $\omega_{2}$ & $\frac{1}{k}$ & 0 \\
& & $\omega_{1}+\omega_{2}$ & $-\frac{1}{k}$ & $\omega_{1}$ \\
\hline $\operatorname{cn} u$ & $\left(2 \omega_{1}, \omega_{1}+2 \omega_{2}\right)$ & $\omega_{2}$ & $-\frac{i}{k}$ & $\frac{\omega_{1}}{2}$ \\
& & $\omega_{1}+\omega_{2}$ & $\frac{i}{k}$ & $\frac{3 \omega_{1}}{2}$ \\
\hline $\operatorname{dn} u$ & $\left(\omega_{1}, 4 \omega_{2}\right)$ & $\omega_{2}$ & $-i$ & $\frac{\omega_{1}}{2}+\omega_{2}$ \\
& & $3 \omega_{2}$ & $i$ & $\frac{\omega_{1}}{2}+3 \omega_{2}$ \\
\hline
\end{tabular}

Table 1: General description of the Jacobian elliptic functions. 
The elliptic functions have the following shift properties.

$$
\operatorname{sn}\left(u+\frac{\omega_{1}}{2}\right)=\frac{\operatorname{cn} u}{\operatorname{dn} u}, \quad \operatorname{cn}\left(u+\frac{\omega_{1}}{2}\right)=-k^{\prime} \frac{\operatorname{sn} u}{\operatorname{dn} u}, \quad \operatorname{dn}\left(u+\frac{\omega_{1}}{2}\right)=k^{\prime} \frac{1}{\operatorname{dn} u} .
$$

We also need the following shift properties of the Jacobian elliptic functions

$$
\begin{aligned}
\operatorname{sn}\left(u+\omega_{1}\right)=-\operatorname{sn}(u), & \operatorname{cn}\left(u+\omega_{1}\right)=-\operatorname{cn}(u), \quad \operatorname{dn}\left(u+\omega_{1}\right)=\operatorname{dn}(u), \\
\operatorname{sn}\left(u+\omega_{2}\right)=\frac{1}{k \operatorname{sn}(u)}, & \operatorname{cn}\left(u+\omega_{2}\right)=-i \frac{\operatorname{dn}(u)}{k \operatorname{sn}(u)}, \quad \operatorname{dn}\left(u+\omega_{2}\right)=-i \frac{\operatorname{cn}(u)}{\operatorname{sn}(u)}, \\
\operatorname{sn}\left(u+\omega_{3}\right)=-\frac{1}{k \operatorname{sn}(u)}, & \operatorname{cn}\left(u+\omega_{3}\right)=i \frac{\operatorname{dn}(u)}{k \operatorname{sn}(u)}, \operatorname{dn}\left(u+\omega_{3}\right)=-i \frac{\operatorname{cn}(u)}{\operatorname{sn}(u)} .
\end{aligned}
$$

where $\omega_{3}=\omega_{1}+\omega_{2}$. The derivatives of the elliptic functions are given by

$$
\begin{aligned}
& \frac{d}{d u} \operatorname{sn} u=\operatorname{cn} u \operatorname{dn} u, \\
& \frac{d}{d u} \operatorname{cn} u=-\operatorname{sn} u \operatorname{dn} u, \\
& \frac{d}{d u} \operatorname{dn} u=-k^{2} \operatorname{sn} u \operatorname{cn} u .
\end{aligned}
$$

The Weierstrass $\wp$ function and related functions

The Weierstrass function can be defined by the following expansion

$$
\wp(z)=\frac{1}{z^{2}}+\sum_{m, n \neq 0}\left(\frac{1}{\left(z-2 m \omega_{1}-2 n \omega_{2}\right)^{2}}-\frac{1}{\left(2 m \omega_{1}-2 n \omega_{2}\right)^{2}}\right) .
$$

It is an even function with periods $\left(2 \omega_{1}, 2 \omega_{2}\right)$. The derivatives of the $\wp$ function vanish at the following points

$$
\wp^{\prime}\left(\omega_{1}\right)=0, \quad \wp^{\prime}\left(\omega_{2}\right)=0, \quad \wp^{\prime}\left(\omega_{1}+\omega_{2}\right)=0 .
$$

The Weierstrass $\wp$ function can be thought of as building blocks of elliptic functions since any elliptic function of period $\left(2 \omega_{1}, 2 \omega_{2}\right)$ can be expresses in terms of the $\wp$ function of the same period. The expression is rational in $\wp(z)$ and linear in $\wp^{\prime}(z)$.

Related to the Weierstrass function is the function $\zeta(z)$ which is defined as

$$
\frac{d \zeta(z)}{d z}=-\wp(z) .
$$

The $\zeta(z)$ function is an odd function of $z$, it is quasi-periodic in the periods $\omega_{1}$ and $\omega_{2}$ with the quasi periods defined as

$$
\zeta\left(z+2 \omega_{1}\right)=\zeta(z)+2 \eta_{1}, \quad \zeta\left(z+2 \omega_{2}\right)=\zeta(z)+2 \eta_{2},
$$


where $\eta_{1}$ and $\eta_{2}$ are given by

$$
\eta_{1}=\zeta\left(\omega_{1}\right), \quad \eta_{2}=\zeta\left(\omega_{2}\right)
$$

They satisfy the Legendre relation

$$
2\left(\eta_{1} \omega_{2}-\eta_{2} \omega_{1}\right)=\pi i
$$

Since the $\zeta(z)$ function is the integral of the $\wp$ function it is clear that it has a simple pole at the origin $z=0$ with residue 1 . Finally we define the function $\sigma(z)$ by the equation

$$
\frac{d}{d z} \log \sigma(z)=\zeta(z)
$$

The $\sigma(z)$ functions is an odd function in $z$, it is useful for integration of the $\zeta(z)$ function and we use it to perform the Strebel integrations. It satisfies the following quasi-periodic relations

$$
\sigma\left(z+2 \omega_{1}\right)=-e^{2 \eta_{1}\left(z+\omega_{1}\right)} \sigma(z), \quad \sigma\left(z+2 \omega_{2}\right)=-e^{2 \eta_{2}\left(z+\omega_{2}\right)} \sigma(z) .
$$

\section{Relationship between elliptic functions and Weierstrass $\wp$ function}

In this paper we extensively use the relationship between the Weierstrass $\wp$ function and the Jacobian elliptic function $\operatorname{sn}(u)$. As mentioned earlier, any doubly periodic function of periods $2 \omega_{1}$ and $2 \omega_{2}$ can always be written in terms of the Weierstrass $\wp$-function having the same periodicity. This is given by

$$
\operatorname{sn}\left(u+\frac{\omega_{1}}{2}\right)=1+\frac{1}{2} \frac{k^{2}-1}{\left(\wp(u)-\frac{5 k^{2}-1}{12}\right)} .
$$

Here we have used the theorm of uniformisation of curves of genus unity given in [55], page 454. The above equation is easily verified for $u=0$. There is another relation one can obtain using the $\wp$ function and the Jacobian elliptic function. Let the values of the $\wp$ at half the periods be given by

$$
\wp\left(\omega_{1}\right)=e_{1}, \quad \wp\left(\omega_{2}\right)=e_{2}, \quad \wp\left(\omega_{3}\right)=e_{3} .
$$

where $\omega_{3}=\omega_{1}+\omega_{2}$. Then from (A.17) it can be shown that

$$
e_{1}=\frac{1+k^{2}}{6}, \quad e_{2}=-\frac{1-6 k+k^{2}}{12}, \quad e_{3}=-\frac{1+6 k+k^{2}}{12} .
$$


We then have the following relation between the $\wp$ function and the sn function.

$$
\wp(u)=e_{3}+\frac{e_{1}-e_{3}}{\operatorname{sn}^{2}\left(u \sqrt{e_{1}-e_{3}}, \tilde{k}\right)} .
$$

Here note that the $\wp$ function is doubly periodic with periods $\left(2 \omega_{1}, 2 \omega_{2}\right)$, and the modulus of the $\operatorname{sn}(u)$ function is given by

$$
\tilde{k}=\sqrt{\frac{e_{2}-e_{3}}{e_{1}-e_{3}}}=2 \frac{\sqrt{k}}{1+k} .
$$

\section{B. Strebel differential in terms of Weierstrass functions}

In this section we will write the general Strebel differential given in (2.3) in two convenient forms. One involves Weierstrass $\wp$ functions and the second involves the $\zeta(u)$ function. The former is useful to obtain relations between the poles of the Strebel differential and the perimeters while the latter is useful to perform the Strebel integration in (2.11) and (2.12).

$\sqrt{\phi(u)}$ in terms of $\wp$-function

It is useful to write the differential $\sqrt{\phi(u)} d u$ in terms of the Weierstrass $\wp$ function for the purpose of obtaining the Strebel lengths by integration as well as to obtain relations between the poles of the Strebel differential and the perimeters. To do this we take the view that we are given the residues $r_{i}$ at the poles $u_{i}$ of the dif-

ferential $\sqrt{\phi(u)}$ in (2.6). It is clear from the transformation between the $z$-plane to the $u$-plane given in (2.5), that the differential $\sqrt{\phi(u)}$ is a doubly periodic function of periods $\left(2 \omega_{1}, 2 \omega_{2}\right)$. Then the unique doubly periodic function of periods $\left(2 \omega_{1}, 2 \omega_{2}\right)$ with the residues $\pm r_{i}$ at $\pm u_{i}$ and a double zero at $u=0$ is given by

$$
\sqrt{\phi(u)}=i \sum_{i=0}^{3} r_{i} \frac{\wp^{\prime}\left(u_{i}\right)}{\wp(u)-\wp\left(u_{i}\right)} .
$$

We also have that the above function must have double zeros at $\omega_{1}, \omega_{2}$ and $\omega_{1}+\omega_{2}$. Demanding that the function in (B.1) has zeros at these points gives the following conditions

$$
\sum_{i=0}^{3} r_{i} \frac{\wp^{\prime}\left(u_{i}\right)}{\wp\left(\omega_{1}\right)-\wp\left(u_{i}\right)}=0
$$




$$
\begin{aligned}
& \sum_{i=0}^{3} r_{i} \frac{\wp^{\prime}\left(u_{i}\right)}{\wp\left(\omega_{2}\right)-\wp\left(u_{i}\right)}=0, \\
& \sum_{i=0}^{3} r_{i} \frac{\wp^{\prime}\left(u_{i}\right)}{\wp\left(\omega_{3}\right)-\wp\left(u_{i}\right)}=0 .
\end{aligned}
$$

where $\omega_{3}=\omega_{1}+\omega_{2}$. It is clear that the derivative of the function $\sqrt{\phi(u)}$ given in $(\mathbb{B} .1)$ at the points $\omega_{1}, \omega_{2}, \omega_{3}$ vanishes since $\wp^{\prime}\left(\omega_{i}\right)=0$. The equations (B.2) together with the fact that the derivative of the function $\sqrt{\phi(u)}$ vanishes at the points $\omega_{1}, \omega_{2}, \omega_{3}$ ensures that the representation of the Strebel differential in terms of the $\wp$ function in (B.1) has double zeros at these points. The three equations in (B.2) enables one to determine the position of the poles $u_{1}, u_{2}, u_{3}$. in terms of $u_{0}$ and the residues $r_{i}$.

\section{Conditions on zeros}

Using the indentity $(\mathrm{A} .20)$ one can cast the equations in $(\mathrm{B} .2)$ entirely in terms of the Jacobian elliptic functions. From (A.20) we can obtain the following useful identities

$$
\begin{aligned}
& \frac{\wp^{\prime}(u)}{\wp\left(\omega_{3}\right)-\wp(u)}=2 \sqrt{e_{1}-e_{3}} \frac{\operatorname{cn}\left(u \sqrt{e_{1}-e_{3}}, \tilde{k}\right) \operatorname{dn}\left(u \sqrt{e_{1}-e_{3}}, \tilde{k}\right)}{\operatorname{sn}\left(u \sqrt{e_{1}-e_{3}}, \tilde{k}\right)}, \\
& \frac{\wp^{\prime}(u)}{\wp\left(\omega_{1}\right)-\wp(u)}=2 \sqrt{e_{1}-e_{3}} \frac{\operatorname{dn}\left(u \sqrt{e_{1}-e_{3}}, \tilde{k}\right)}{\operatorname{cn}\left(u \sqrt{e_{1}-e_{3}}, \tilde{k}\right) \operatorname{sn}\left(u \sqrt{e_{1}-e_{3}}, \tilde{k}\right)}, \\
& \frac{\wp^{\prime}(u)}{\wp\left(\omega_{2}\right)-\wp(u)}=2 \sqrt{e_{1}-e_{3}} \frac{\operatorname{cn}\left(u \sqrt{e_{1}-e_{3}}, \tilde{k}\right)}{\operatorname{dn}\left(u \sqrt{e_{1}-e_{3}}, \tilde{k}\right) \operatorname{sn}\left(u \sqrt{e_{1}-e_{3}}, \tilde{k}\right)} .
\end{aligned}
$$

Substituting the above identities in (B.2) it is easy to show using simple manipulations and indentities involving Jacobian elliptic functions, that the conditions reduce to the following three equations

$$
\begin{aligned}
& \sum_{i} r_{i} \frac{\operatorname{dn}\left(2 u \sqrt{e_{1}-e_{3}}, \tilde{k}\right)}{\operatorname{sn}\left(2 u \sqrt{e_{1}-e_{3}}, \tilde{k}\right)}=0, \\
& \sum_{i} r_{i} \frac{\operatorname{cn}\left(2 u \sqrt{e_{1}-e_{3}}, \tilde{k}\right)}{\operatorname{sn}\left(2 u \sqrt{e_{1}-e_{3}}, \tilde{k}\right)}=0, \\
& \sum_{i} r_{i} \frac{1}{\operatorname{sn}\left(2 u \sqrt{e_{1}-e_{3}}, \tilde{k}\right)}=0 .
\end{aligned}
$$

We now use the following Landen transformations [55] to convert the Jacobi Elliptic functions with modulus $\tilde{k}$ to that with modulus $k$.

$$
\operatorname{sn}((1+k) u, \tilde{k})=(1+k) \frac{\operatorname{sn}(u, k)}{1+k \operatorname{sn}^{2}(u, k)},
$$




$$
\begin{aligned}
\operatorname{cn}((1+k) u, \tilde{k}) & =\frac{\operatorname{cn}(u, k) \operatorname{dn}(u, k)}{1+k \operatorname{sn}^{2}(u, k)}, \\
\operatorname{dn}((1+k) u, \tilde{k}) & =\frac{1-k \operatorname{sn}^{2}(u, k)}{1+k \operatorname{sn}^{2}(u, k)}
\end{aligned}
$$

Note that the argument involving $u$ which occurs in $(\mathrm{B} .4)$ is $2 u \sqrt{e_{1}-e_{3}}=(1+k) u$. Substituting the above transformations in (B.4) and after some simple manipulations we obtain the following equivalent conditions which enable us to determine $u_{1}, u_{2}, u_{3}$ in terms of $u_{0}$ and $r_{i}$.

$$
\begin{aligned}
\sum_{i} r_{i} \frac{1}{\operatorname{sn}\left(u_{i}\right)} & =0, \\
\sum_{i} r_{i} \operatorname{sn}\left(u_{i}\right) & =0, \\
\sum_{i} r_{i} \frac{\operatorname{cn}\left(u_{i}\right) \operatorname{dn}\left(u_{i}\right)}{\operatorname{sn}\left(u_{i}\right)} & =0 .
\end{aligned}
$$

As a simple consistency check we can show that the residues given in (2.7) satisfy the equations (B.6). Substituting the values of $r_{i}$ in (B.6) and after a few manipulations, the three set of equations reduce to

$$
\begin{aligned}
& \sum_{i} \frac{1}{\prod_{j \neq i}\left(z_{i}-z_{j}\right)}=0, \\
& \sum_{i} \frac{z_{i}}{\prod_{j \neq i}\left(z_{i}-z_{j}\right)}=0, \\
& \sum_{i} \frac{z_{i}^{2}}{\prod_{j \neq i}\left(z_{i}-z_{j}\right)}=0,
\end{aligned}
$$

here $z_{i}=\operatorname{cn}\left(u_{i}\right) / d n\left(u_{i}\right)$. It can be shown by simple algebra that the above set of equations are identities for any set of $z_{i}^{\prime} s$. Thus we have verified the conditions for the zeros (B.6)

The Strebel differential in terms of the the $\zeta(u)$ function and the Strebel lengths

We now show that the Strebel differential $\sqrt{\phi(u)} d u$ can also be written in terms of the $\zeta(u)$ function. This allows one to perform the Strebel integral and obtain the Strebel lengths. From (B.1) we see that Strebel differential is expressed as a linear combination of the function

$$
i r_{i} \frac{\wp^{\prime}\left(u_{i}\right)}{\wp(u)-\wp\left(u_{i}\right)}
$$


This is periodic function with periods $\left(2 \omega_{1}, 2 \omega_{2}\right)$, with double zeros at the origin $u=0$ and poles at $u= \pm u_{i}$ with residues $\pm r_{i}$. From the properties of the $\zeta(u)$ function we see that the function in (B.8) can be written as

$$
i r_{i} \frac{\wp^{\prime}\left(u_{i}\right)}{\wp(u)-\wp\left(u_{i}\right)}=i r_{i}\left(\zeta\left(u+u_{i}\right)-\zeta\left(u-u_{i}\right)-2 \zeta\left(u_{i}\right)\right) .
$$

We now show the above indentity is true by matching the singularity structure on both sides of the equation. Firstly, from (A.12) we see that the right hand side of the above equation is also periodic with periods $\left(2 \omega_{1}, 2 \omega_{2}\right)$. Since the function $\zeta(u)$ has a simple pole at $u=0$ with residue 1 , the above combination of $\zeta$ functions has simple poles at $u= \pm u_{i}$ with residues $\pm r_{i}$. Furthermore $\zeta(u)$ is an even function, which implies both sides of the equation vanish at $u=0$.Finally, since the derivative of the $\zeta(u)$ function is the $\wp(u)$ function which is even in $u$, the derivative of the right hand side vanishes at $u=0$. implying $u=0$ is a double zero. This establishes the indentity in (B.9). Substituting (B.9) in (B.1) we obtain

$$
\sqrt{\phi(u)} d u=i \sum_{i} r_{i}\left(\zeta\left(u+u_{i}\right)-\zeta\left(u-u_{i}\right)-2 \zeta\left(u_{i}\right)\right) d u .
$$

To perform the Strebel integrals in (2.11) and (2.12) we write the above equation in terms of the $\sigma$ functions using (A.15). This gives

$$
\sqrt{\phi(u)} d u=i \sum_{i} r_{i}\left(\frac{d}{d u} \log \sigma\left(u+u_{i}\right)-\frac{d}{d u} \log \sigma\left(u-u_{i}\right)-2 \zeta\left(u_{i}\right)\right) d u .
$$

It is now easy to perform the integrals from 0 to $\omega_{1}$ and 0 to $\omega_{2}$ giving

$$
\begin{aligned}
a & =\sum_{i} r_{i}\left[\pi-2 i\left(\zeta\left(u_{i}\right) \omega_{1}-\zeta\left(\omega_{1}\right) u_{i}\right)\right], \\
b & =\sum_{i} r_{i}\left[\pi+2 i\left(\zeta\left(u_{i}\right) \omega_{2}-\zeta\left(\omega_{2}\right) u_{i}\right)\right] .
\end{aligned}
$$

Here we have used the quasi-periodic relations of the $\sigma(u)$ function given in (A.16)

\section{The case of equal perimeters}

The case of equal perimeters $r_{0}=r_{1}=r_{2}=r_{3}=r$ has been studied in [56]. Here we show that we can solve the conditions on the zeros (B.6) easily when the perimeters are equal, and the equations for the Stebel lengths $(\mathbb{B} .12)$ reduce to the 
equations found by [56]. For the case of equal perimeters the conditions on the zeros reduce to

$$
\sum_{i} \frac{1}{\operatorname{sn}\left(u_{i}\right)}=0, \quad \sum_{i} \operatorname{sn}\left(u_{i}\right)=0, \quad \sum_{i} \frac{\operatorname{cn}\left(u_{i}\right) \operatorname{dn}\left(u_{i}\right)}{\operatorname{sn}\left(u_{i}\right)}=0 .
$$

These conditions are satisfied by the choice

$$
u_{0}, \quad u_{1}=u_{0}+\omega_{1}, \quad u_{2}=u_{0}+\omega_{2}, \quad u_{3}=u_{0}+\omega_{3}
$$

Subtituting these values of $u_{i}$ in the conditions (B.13), it is easily seen that they are satisfied using the properties in (A.7). Writing out the Strebel differential $\sqrt{\phi(u)}$ using the above solution for $u_{i}$ we obtain

$$
\begin{aligned}
\sqrt{\phi(u)} d u & =i r\left(\frac{\wp^{\prime}\left(u_{0}\right)}{\wp(u)-\wp\left(u_{0}\right)}+\frac{\wp^{\prime}\left(u_{0}+\omega_{1}\right)}{\wp(u)-\wp\left(u_{0}+\omega_{1}\right)}\right. \\
& \left.+\frac{\wp^{\prime}\left(u_{0}+\omega_{2}\right)}{\wp(u)-\wp\left(u_{0}+\omega_{2}\right)}+\frac{\wp^{\prime}\left(u_{0}+\omega_{3}\right)}{\wp(u)-\wp\left(u_{0}+\omega_{3}\right)}\right) .
\end{aligned}
$$

It is clear from the above expression that the Strebel differential for this case has a periodicity with smaller periods $\left(\omega_{1}, \omega_{2}\right)$ as found in [56] Using this fact we can rewrite the differential in terms of the Weierstrass $\wp$ function with smaller periods, this leads to the following expression

$$
\sqrt{\phi(u)} d u=\operatorname{ir}\left(\frac{\tilde{\wp}\left(u_{0}\right)}{\tilde{\wp}(u)-\tilde{\wp}\left(u_{0}\right)}\right)
$$

here $\tilde{\wp}(u)$ is the Weierstrass function with periods $\left(\omega_{1}, \omega_{2}\right)$. From the above expression one can proceed with the remaining analysis to obtain the Strebel lengths $a, b$ as discussed here to obtain the equations for the Strebel lengths found by [56].

\section{The elementary derivation of equation (3.12)}

A Strebel differential with a fourth order zero can be written as

$$
\phi(z) d z^{2}=C \frac{\left(z-z_{0}\right)^{4} d z^{2}}{z^{2}(z-1)^{2}(z-\eta)^{2}},
$$

where we have chosen to put the poles at $0,1, \infty$ and $\eta$ using $S L(2, C)$ invariance. Labelling the positive residues at these poles as $\left(p_{1}, p_{2}, p_{3}\right)$ and $p_{0}$ respectively, we see that

$$
\frac{p_{1}}{p_{3}}=-\frac{z_{0}^{2}}{\eta} \quad \frac{p_{2}}{p_{3}}=-\frac{\left(z_{0}-1\right)^{2}}{1-\eta}
$$


We also have $p_{0}=p_{1}+p_{2}+p_{3}$. We can eliminate $z_{0}$ from the two equations in (C.2) getting the quadratic equation for $\eta$

$$
\left(p_{1}+p_{2}\right) \eta+2 \sqrt{-p_{1} p_{3}} \sqrt{\eta}-\left(p_{2}+p_{3}\right)
$$

The solution to this quadratic equation gives

$$
\sqrt{\eta}=\left(\frac{\sqrt{p_{0} p_{2}} \pm i \sqrt{p_{1} p_{3}}}{p_{1}+p_{2}}\right),
$$

which is the same as (3.12)

To connect with the expressions in [43] for extremal correlators, we need to take $p_{0}=p_{t}, p_{1}=p_{-t}, p_{2}=p_{\infty}, p_{3}=p_{1}$, where the $p$ 's on the left hand side are ours and those on the right are theirs. Their quantity $t$ is related to $\eta$ by

$$
\eta=\frac{(t-(-t))(1-\infty)}{(t-1)(-t-\infty)}=\frac{2 t}{t-1}
$$

According to 43 the quadratic equation which determines $t$ is given by

$$
\begin{array}{r}
t^{2}\left(4+B^{2}+4 A\right)+2 t(2 B+A B)+A^{2}=0 \quad \text { where } \\
A=\frac{p_{-t}}{p_{\infty}}-\frac{p_{t}}{p_{\infty}}, \quad B=\frac{p_{t}}{p_{\infty}}+\frac{p_{-t}}{p_{\infty}}
\end{array}
$$

Subtituting for $t$ in terms of $\eta$ in the above quadriatic equation we obtain the following equation for $\eta$

$$
\left(p_{\infty}+p_{-t}\right) \eta^{2}-2 \eta\left(p_{\infty} p_{t}-p_{1} p_{-t}\right)+\left(p_{t}-p_{-t}\right)^{2}=0
$$

The two solutions of this equation are given in (3.12) after making the above identifications.

\section{Details on the approximation scheme}

In this appendix we provide the details leading to the equations (5.1) and (5.3) which are the basic equations to obtain a perturbation scheme around the Y-diagram.

We start out with the conditions on the zeros given in (2.9) and perform a large $z$ expansion of these equations. As shown in (3.3) large $z$ corresponds to expansion aroudn $\bar{u}=\omega_{1} / 2+\omega_{2}$. From the definition of $z=\operatorname{cn} u / \operatorname{dn} u$ in (2.5) and using the 
identities in (A.2) we can obtain the following large $z$ expansion of the Jacobian elliptic functions

$$
\begin{aligned}
\operatorname{sn} u & =\frac{1}{k}\left(1+\frac{1}{2}\left(\frac{1}{k^{2}}-1\right) \frac{\epsilon^{2}}{z^{\prime 2}}+\left(\frac{3}{8 k^{4}}-\frac{1}{4 k^{2}}-\frac{1}{8}\right) \frac{\epsilon^{4}}{z^{\prime 4}}+\cdots\right), \\
\operatorname{cn} u & =\frac{\sqrt{k^{2}-1}}{k}\left(1+\frac{\epsilon^{2}}{2 k^{2} z^{\prime 2}}+\frac{3 \epsilon^{4}}{8 k^{4} z^{\prime 4}}+\cdots\right), \\
\operatorname{dn} u & =\frac{\epsilon}{z^{\prime}} \operatorname{cn} u, \\
\frac{\operatorname{cn} u \operatorname{dn} u}{\operatorname{sn} u} & =\frac{1}{k}\left(\left(k^{2}-1\right) \frac{\epsilon}{z^{\prime}}+\left(\frac{k^{2}}{2}-\frac{1}{2 k^{2}}\right) \frac{\epsilon^{3}}{z^{\prime 3}}+\cdots\right),
\end{aligned}
$$

here we have substituted $z=z^{\prime} / \epsilon$ with $z^{\prime}$ finite to organize the expansion in powers of $\epsilon$. Substituting these expansions for the Jacobian elliptic functions that occur in the conditions for the zeros (2.9) with $z_{i}^{\prime}=1 / x_{i}$ we obtain

$$
\begin{aligned}
\sum_{i=0}^{3} r_{i}\left(1-\frac{1}{2}\left(\frac{1}{k^{2}}-1\right) \epsilon^{2} x_{i}^{2}+\left(\frac{-1}{8 k^{4}}-\frac{1}{4 k^{2}}+\frac{3}{8}\right) \epsilon^{4} x_{i}^{4}+\cdots\right) & =0 \\
\sum_{i=0}^{3} r_{i}\left(1+\frac{1}{2}\left(\frac{1}{k^{2}}-1\right) \epsilon^{2} x_{i}^{2}+\left(\frac{3}{8 k^{4}}-\frac{1}{4 k^{2}}-\frac{1}{8}\right) \epsilon^{4} x_{i}^{4}+\cdots\right) & =0 \\
\sum_{i=0}^{3} r_{i}\left(\left(k^{2}-1\right) \epsilon x_{i}+\frac{1}{2 k^{2}}\left(k^{4}-1\right) \epsilon^{3} x_{i}^{3}\right) & =0 .
\end{aligned}
$$

Adding and subtracting the first two equations and simplifying the third equation we obtain

$$
\begin{aligned}
\sum_{i=0}^{3} r_{i}+\sum_{i=0}^{3} r_{i}\left(1-k^{2}\right)^{2} \frac{\epsilon^{4} x_{i}^{4}}{8 k^{4}} & =0 \\
\sum_{i=0}^{3} r_{i}\left(x_{i}^{2}+\frac{1}{2}\left(1+\frac{1}{k^{2}}\right) \epsilon^{2} x_{i}^{4}\right) & =0 \\
\sum_{i=0}^{3} r_{i}\left(x_{i}+\frac{1}{2}\left(1+\frac{1}{k^{2}}\right) \epsilon^{2} x_{i}^{3}\right) & =0 .
\end{aligned}
$$

Note that we have retained only the leading two terms in each of the equation. The above equations are the leading conditions on the positions of the zeros which now correspond to $x_{i}$. For later convenience we define the following quantities

$$
\tilde{E}=-\frac{\left(1-k^{2}\right)^{2}}{8 k^{4}}, \quad E=-\frac{1}{2}\left(1+\frac{1}{k^{2}}\right) .
$$

We now find the leading expansions of the equation for the Strebel length $a$ in (2.14) and the linear combination of lengths in (2.14) around $\bar{u}$. Expanding the 
equation for the Strebel length $a$ around $\bar{u}$ we obtain

$$
a=A_{0} \sum r_{i}+\epsilon A_{1} \sum r_{i} x_{i}+\epsilon^{2} A_{2} \sum r_{i} x_{i}^{2}+\epsilon^{3} A_{3} \sum r_{i} x_{i}^{3}+\epsilon^{4} A_{4} \sum r_{i} x_{i}^{4},
$$

where

$$
\begin{aligned}
& A_{0}=\pi-2 i\left(\zeta(\bar{u}) \omega_{1}-\zeta\left(\omega_{1}\right) \bar{u}\right), \quad A_{1}=\frac{2 i}{k}\left(\zeta^{(1)}(\bar{u}) \omega_{1}-\zeta\left(\omega_{1}\right)\right), \\
& A_{2}=-\frac{2 i}{2 ! k^{2}} \zeta^{(2)}(\bar{u}) \omega_{1}, \quad A_{3}=2 i\left(\zeta^{(1)}(\bar{u}) \omega_{1}-\zeta\left(\omega_{1}\right)\right) \frac{1}{6 k}\left(1+\frac{1}{k^{2}}\right)+\frac{2 i}{3 ! k^{3}} \zeta^{(3)}(\bar{u}) \omega_{1}, \\
& A_{4}=-\frac{i}{3 k^{2}}\left(1+\frac{1}{k^{2}}\right) \zeta^{(2)}(\bar{u}) \omega_{1}-\frac{2 i}{4 ! k^{4}} \zeta^{(4)}(\bar{u}) \omega_{1} .
\end{aligned}
$$

here the summations $\sum$ runs from 0 to 3 , and

$$
\zeta^{(n)}(\bar{u})=\left.\frac{d^{n}}{d u^{n}} \zeta(u)\right|_{u=\bar{u}} .
$$

To obtain the above expansion for $a$ we have performed a taylor series expansion of the equation for the Strebel length $a$ in (2.14) about the point $\bar{u}$ and then substituted for $u_{i}$ in terms of $x_{i}$ using (3.1). Note that all the coefficients $A_{n}$ start at $O\left(\epsilon^{0}\right)$. Performing a similar expansion in (2.15) and retaining terms till order $\epsilon^{4}$ we obtain

$$
\begin{aligned}
a \omega_{2}+b \omega_{1} & =B_{0} \sum r_{i}+\epsilon B_{1} \sum r_{i} x_{i}+\epsilon^{3} B_{3} \sum r_{i} x_{i}^{3}, \\
\text { with } B_{0} & =\pi \frac{\omega_{1}}{2}, \quad B_{1}=\frac{\pi}{k}, \quad B_{3}=\frac{\pi}{6 k}\left(1+\frac{1}{k^{2}}\right) .
\end{aligned}
$$

Here again the $B_{n}$ 's start of at $O\left(\epsilon^{0}\right)$. We now can eliminate the combination $\sum r_{i}$, $\sum r_{i} x_{i}$ and $\sum r_{i} x_{i}^{2}$ using $(\underline{D .3})$ in (D.5) and (D.8) to obtain the following pairs of equations for the Strebel lengths $a$ and $b$

$$
\begin{aligned}
a & =\epsilon^{3} p_{1} \sum r_{i} x_{i}^{3}+\epsilon^{4} p_{2} \sum r_{i} x_{i}^{4}, \\
a \omega_{2}+b \omega_{1} & =\epsilon^{3} q_{1} \sum r_{i} x_{i}^{3}+\epsilon^{4} q_{2} \sum r_{i} x_{i}^{4} .
\end{aligned}
$$

with

$$
\begin{array}{r}
p_{1}=A_{1} E+A_{3}, \quad p_{2}=A_{0} \tilde{E}+A_{2} E+A_{4}, \\
q_{1}=B_{1} E+B_{3}, \quad q_{2}=B_{0} \tilde{E} .
\end{array}
$$

From (D.9) it is clear that the Strebel lengths $a, b$ begin at $O\left(\epsilon^{3}\right)$. This fact can also be easily verified by performing the scaling $z=z^{\prime} / \epsilon$ in the basic equation for the Strebel differential (2.3). 
From the discussion in section 5., we see that among the constants in (D.10) the relevant quantity is the ratio $p_{1} / q_{1}$. Here we evaluate this ratio, from the definition of $p_{1}$ in terms of $A_{1}$ and $A_{3}$ we see that we we first need to evaluate the derivatives $\zeta^{(1)}(\bar{u})$ and $\zeta^{(3)}(\bar{u})$. Using the defintion of $\zeta$ in (A.11), we have

$$
\begin{aligned}
\zeta^{(1)}(u) & =-\wp(u) \\
& =\frac{1-5 k^{2}}{12}+\frac{1-k^{2}}{2} \frac{\operatorname{dn} u}{\operatorname{cn} u-\operatorname{dn} u},
\end{aligned}
$$

here we have used (A.17) to re-write the Weierstrass $\wp$ function in terms of the elliptic functions. From table 1. we see that the elliptic function $\operatorname{dn}(u)$ vanishes at $\bar{u}=\omega_{1} / 2+\omega_{2}$, therefore we obtain

$$
\zeta^{(1)}(\bar{u})=\frac{1-5 k^{2}}{12}
$$

Differentiating (D.11) twice we obtain

$$
\zeta^{(3)}(u)=\frac{\left(1-k^{2}\right)^{2}}{2} \frac{\operatorname{dn} u\left(1+\operatorname{sn}^{2} u\right)-\operatorname{cn} u\left(1+k^{2} \operatorname{sn}^{2} u\right)}{(\operatorname{cn} u-\operatorname{dn} u)^{3}},
$$

again since $\operatorname{dn}(\bar{u})=0$ we get

$$
\begin{aligned}
\zeta^{(3)}(\bar{u}) & =-\frac{\left(1-k^{2}\right)^{2}\left(1+k^{2} \operatorname{sn}^{2} \bar{u}\right)}{2 \operatorname{cn}^{2} \bar{u}} \\
& =\left(1-k^{2}\right) k^{2}
\end{aligned}
$$

where we have substituted the values of the Jacobi elliptic functions at $\bar{u}$ from (A.6) and table 1 . Now we can evaluate the ratio $p_{1} / q_{1}$, using the defintions (D.10), (D.6) and (5.2) and the equations (D.12) and (D.14) we see that

$$
\begin{aligned}
\frac{p_{1}}{q_{1}} & =\frac{2 i}{\pi}\left(\frac{1-10 k^{2}+k^{4}}{12} \omega_{1}-\zeta\left(\omega_{1}\right)\right) \\
& =2 i\left(\frac{1-10 k^{2}+k^{4}}{12} \vartheta_{3}^{2}(q)+\frac{1}{12} \frac{1}{\vartheta_{3}^{2}(q)} \frac{\vartheta_{1}^{\prime \prime \prime}(q)}{\vartheta_{1}^{\prime}(q)}\right) .
\end{aligned}
$$

To obtain the second line in the above equation we have used the relations 55

$$
\omega_{1}=\pi \vartheta_{3}^{2}(q), \quad \zeta\left(\omega_{1}\right)=-\frac{\pi^{2}}{12 \omega_{1}} \frac{\vartheta_{1}^{\prime \prime \prime}(q)}{\vartheta_{1}^{\prime}(q)} .
$$

In all these equations $q=\exp \left(2 \pi i \omega_{2} / \omega_{1}\right)$, which implies

$$
\frac{\omega_{2}}{\omega_{1}}=\frac{1}{2 \pi i} \log (q)
$$

Finally, the modulus $k$ can also be written in terms of $q$ by the following equation 55

$$
k=\frac{\vartheta_{2}^{2}(q)}{\vartheta_{3}^{2}(q)}
$$




\section{E. Solution for the modulus $k$ in terms of the Strebel lengths.}

In this section we solve the following equation modulus $k$ in terms of the Strebel lengths $a^{(3)}$ and $b^{(3)}$.

$$
\frac{\omega_{1} p_{1}}{q_{1}}\left(\frac{\omega_{2}}{\omega_{1}}+\frac{1}{\rho}\right)=1
$$

The above equation is basically a simple re-writing of the equation (5.8). From now on we drop the superscript ${ }^{(0)}$ which indicates the zeroth order terms, $\rho=a^{(3)} / b^{(3)}$. The strategy we use is to first write all the quantities that occur in the above equation in terms of the modular parameter $q$ in (D.17), use (E.1) to solve for $q$ in terms of the ratio of Strebel lengths $\rho$ and then write $k$ in terms of $\rho$ using (D.18). Since the functions involved in the equation (E.1) are transcendental in $q$, we need to perform an expansion in $q$ to solve for $q$ in terms of $q$. This assumes that $|q|<1$, and for this expansion to be consistent it will turn out that $\rho<1$. Substituting the expansions of the theta functions that occur in (D.15), (D.16) and,(D.18) we obtain the following expansion

$$
\frac{\omega_{1} p_{1}}{q_{1}}=2 \pi i\left(-14 q+260 q^{2}-6200 q^{3}+143368 q^{4}+\cdots\right) .
$$

Using this expansion in (E.1) we obtain

$$
\begin{aligned}
\rho & =\frac{\omega_{1} p_{1}}{q_{1}}\left(1-\frac{\omega_{1} p_{1}}{q_{1}} \frac{\log q}{2 \pi i}\right)^{-1}, \\
& =-28 i \pi q+392 \pi i q^{2} \log q+520 \pi i q^{2}+\cdots .
\end{aligned}
$$

We can now invert this equation and obtain $q$ in terms of $\rho$, which is given by

$$
q=\frac{i}{28 \pi} \rho-\frac{1}{56 \pi^{2}} \rho^{2} \log \rho-\frac{1}{2749 \pi^{2}}\left(65-49 \log (28 \pi)+i \frac{\pi}{2}\right) \rho^{2}+\cdots .
$$

It is clear from this solution it is consistent to assume $|q|<1$. From this the leading order solution to the modulus $k$ in terms of the ratio $\rho$ is given by

$$
k=\frac{2 e^{\frac{i \pi}{4}}}{\sqrt{7 \pi}} \sqrt{\rho}(1+O(\rho \log (\rho))) .
$$

It is clear from the approach that one can obtain the solution of $k$ in terms of $\rho$ to any accuracy that is desired. 


\section{References}

[1] J. M. Maldacena, "The large $N$ limit of superconformal field theories and supergravity," Adv. Theor. Math. Phys. 2 (1998) 231-252, hep-th/9711200.

[2] S. S. Gubser, I. R. Klebanov, and A. M. Polyakov, "Gauge theory correlators from non-critical string theory," Phys. Lett. B428 (1998) 105-114, hep-th/9802109.

[3] E. Witten, "Anti-de Sitter space and holography," Adv. Theor. Math. Phys. 2 (1998) 253-291, hep-th/9802150.

[4] R. R. Metsaev and A. A. Tseytlin, "Type IIB superstring action in $A d S_{5} \times S^{5}$ background," Nucl. Phys. B533 (1998) 109-126, hep-th/9805028.

[5] R. R. Metsaev and A. A. Tseytlin, "Superstring action in $A d S_{5} \times S^{5}$ : kappa-symmetry light cone gauge," Phys. Rev. D63 (2001) 046002, hep-th/0007036.

[6] R. R. Metsaev, C. B. Thorn, and A. A. Tseytlin, "Light-cone superstring in AdS space-time," Nucl. Phys. B596 (2001) 151-184, hep-th/0009171.

[7] N. Berkovits, "Super-poincare covariant quantization of the superstring," JHEP 04 (2000) 018, hep-th/0001035.

[8] N. Berkovits and O. Chandia, "Superstring vertex operators in an $A d S_{5} \times S^{5}$ background," Nucl. Phys. B596 (2001) 185-196, hep-th/0009168.

[9] K. Bardakci and C. B. Thorn, "A worldsheet description of large $N_{c}$ quantum field theory," Nucl. Phys. B626 (2002) 287-306, hep-th/0110301.

[10] C. B. Thorn, "A worldsheet description of planar Yang-Mills theory," Nucl. Phys. B637 (2002) 272-292, hep-th/0203167.

[11] K. Bardakci and C. B. Thorn, "A mean field approximation to the world sheet model of planar $\phi^{3}$ field theory," Nucl. Phys. B652 (2003) 196-228, hep-th/0206205.

[12] S. Gudmundsson, C. B. Thorn, and T. A. Tran, "BT worldsheet for supersymmetric gauge theories," Nucl. Phys. B649 (2003) 3-38, hep-th/0209102.

[13] C. B. Thorn and T. A. Tran, "The fishnet as anti-ferromagnetic phase of worldsheet Ising spins," Nucl. Phys. B677 (2004) 289-353, hep-th/0307203.

[14] K. Bardakci, "Further results about field theory on the world sheet and string formation," Nucl. Phys. B715 (2005) 141-172, hep-th/0501107.

[15] P. Haggi-Mani and B. Sundborg, "Free large $N$ supersymmetric Yang-Mills theory as a string theory," JHEP 04 (2000) 031, hep-th/0002189.

[16] H. L. Verlinde, "Bits, matrices and 1/N," JHEP 12 (2003) 052, hep-th/0206059.

[17] J.-G. Zhou, "pp-wave string interactions from string bit model," Phys. Rev. D67 (2003) 026010, hep-th/0208232. 
[18] D. Vaman and H. L. Verlinde, "Bit strings from $N=4$ gauge theory," JHEP 11 (2003) 041, hep-th/0209215.

[19] A. Dhar, G. Mandal, and S. R. Wadia, "String bits in small radius AdS and weakly coupled $N=4$ super Yang-Mills theory. I," hep-th/0304062.

[20] K. Okuyama and L.-S. Tseng, "Three-point functions in $N=4$ SYM theory at one-loop," JHEP 08 (2004) 055, hep-th/0404190.

[21] L. F. Alday, J. R. David, E. Gava, and K. S. Narain, "Structure constants of planar $N=4$ Yang Mills at one loop," JHEP 09 (2005) 070, hep-th/0502186.

[22] J. Engquist and P. Sundell, "Brane partons and singleton strings," hep-th/0508124.

[23] L. F. Alday, J. R. David, E. Gava, and K. S. Narain, "Towards a string bit formulation of $N=4$ super Yang- Mills," hep-th/0510264.

[24] A. Karch, "Lightcone quantization of string theory duals of free field theories," hep-th/0212041.

[25] A. Clark, A. Karch, P. Kovtun, and D. Yamada, "Construction of bosonic string theory on infinitely curved anti-de Sitter space," Phys. Rev. D68 (2003) 066011, hep-th/0304107.

[26] G. Bonelli, "On the boundary gauge dual of closed tensionless free strings in $A d S$," JHEP 11 (2004) 059, hep-th/0407144.

[27] M. Bianchi, J. F. Morales, and H. Samtleben, "On stringy $A d S_{5} \times S^{5}$ and higher spin holography," JHEP 07 (2003) 062, hep-th/0305052.

[28] N. Beisert, M. Bianchi, J. F. Morales, and H. Samtleben, "On the spectrum of AdS/CFT beyond supergravity," JHEP 02 (2004) 001, hep-th/0310292.

[29] J. Teschner, "Liouville theory revisited," Class. Quant. Grav. 18 (2001) R153-R222, hep-th/0104158.

[30] H. Dorn and H. J. Otto, "Two and three point functions in Liouville theory," Nucl. Phys. B429 (1994) 375-388, hep-th/9403141.

[31] A. B. Zamolodchikov and A. B. Zamolodchikov, "Structure constants and conformal bootstrap in Liouville field theory," Nucl. Phys. B477 (1996) 577-605, hep-th/9506136.

[32] J. Teschner, "On the Liouville three point function," Phys. Lett. B363 (1995) 65-70, hep-th/9507109.

[33] A. Pakman, "Liouville theory without an action," hep-th/0601197.

[34] R. Gopakumar, "From free fields to AdS," Phys. Rev. D70 (2004) 025009, hep-th/0308184. 
[35] R. Gopakumar, "From free fields to AdS. II," Phys. Rev. D70 (2004) 025010, hep-th/0402063.

[36] R. Gopakumar, "Free field theory as a string theory?," Comptes Rendus Physique 5 (2004) 1111-1119, hep-th/0409233.

[37] R. Gopakumar, "From free fields to AdS. III," Phys. Rev. D72 (2005) 066008, hep-th/0504229.

[38] M. Carfora, C. Dappiaggi, and V. Gili, "Simplicial aspects of string dualities," AIP Conf. Proc. 751 (2005) 182-184, hep-th/0410006.

[39] E. T. Akhmedov, "Expansion in Feynman graphs as simplicial string theory," JETP Lett. 80 (2004) 218-225, hep-th/0407018.

[40] S. Mamedov and S. Parvizi, "AdS/CFT correspondence via $R$-current correlation functions revisited," hep-th/0505162.

[41] K. Furuuchi, "From free fields to AdS: Thermal case," Phys. Rev. D72 (2005) 066009, hep-th/0505148.

[42] V. L. Gili, "Simplicial and modular aspects of string dualities," hep-th/0605053.

[43] O. Aharony, Z. Komargodski, and S. S. Razamat, "On the worldsheet theories of strings dual to free large $N$ gauge theories," hep-th/0602226.

[44] E. D'Hoker, D. Z. Freedman, S. D. Mathur, A. Matusis, and L. Rastelli, "Extremal correlators in the AdS/CFT correspondence," hep-th/9908160.

[45] O. Aharony, S. S. Gubser, J. M. Maldacena, H. Ooguri, and Y. Oz, "Large $N$ field theories, string theory and gravity," Phys. Rept. 323 (2000) 183-386, hep-th/9905111.

[46] E. D'Hoker and D. Z. Freedman, "Supersymmetric gauge theories and the AdS/CFT correspondence," hep-th/0201253.

[47] S.-M. Lee, S. Minwalla, M. Rangamani, and N. Seiberg, "Three-point functions of chiral operators in $D=4, N=4$ SYM at large $N$," Adv. Theor. Math. Phys. 2 (1998) 697-718, hep-th/9806074.

[48] O. Aharony, Z. Komargodski, and S. S. Razamat. Work in progress.

[49] P. Di Francesco, P. Mathieu, and D. Senechal, "Conformal field theory,". New York, USA: Springer (1997) 890 p.

[50] C. M. Hull, "Lectures on $W$ gravity, $W$ geometry and $W$ strings," hep-th/9302110.

[51] P. C. West, "A review of $W$ strings," hep-th/9309095.

[52] C. N. Pope, "W strings 93," hep-th/9309125. 
[53] M. D. Freeman and P. C. West, "W $W_{3}$ string scattering," Phys. Lett. B299 (1993) 30-36, hep-th/9210134.

[54] S. L. Shatashvili and C. Vafa, "Superstrings and manifold of exceptional holonomy," Selecta Math. 1 (1995) 347, hep-th/9407025.

[55] E. Whittaker and G. Watson, "A course of modern analysis,". Cambridge, UK: Cambridge University Press (1969) 608 p.

[56] A. Belopolsky, "Effective Tachyonic potential in closed string field theory," Nucl. Phys. B448 (1995) 245-276, hep-th/9412106. 